\title{
The ameliorating role of vitamin $E$ against iron overload-induced oxidative stress in rats
}

\author{
Shadia A. Fathi ${ }^{1}$, Ahmed M. Salem ${ }^{1}$, El Sayed A. Hassanin ${ }^{2}$, Mohamed A. M. Ali ${ }^{1}$ and Amr A. E. \\ Goma $^{2 *}$ \\ ${ }^{1}$ Department of Biochemistry, Faculty of science, Ain Shams University, Cairo, Egypt \\ ${ }^{2}$ National Nutrition Institute, Cairo, Egypt
}

\section{A R T ICLE INFO}

Article history:

Received 18 July 2016

Accepted 22 September 2016

Keywords:

Iron overload;

Oxidative stress;

Vitamin E;

Antioxidant activity.

\begin{abstract}
A B S T R A C T
Iron is an essential metal involved in a wide spectrum of physiological functions. However, excess iron is toxic because it promotes the generation of free radicals, which lead to oxidative stress and oxidative tissue damage. Vitamin $\mathrm{E}$ is the most potent liposoluble antioxidant and has the potential to improve tolerance of iron supplementation and prevent further tissue damage. The present study aimed to investigate the effects of different levels of iron supplementation with or without vitamin $\mathrm{E}$ on body weight gain, food intake, feed efficiency ratio, relative organs weight, hemoglobin concentration, hematocrit level, iron profile, liver function, lipid profile, lipid peroxidation level, antioxidant enzymes activity and histological architecture of liver tissues in male albino rats. Seventy-two rats were randomly divided into nine groups (8 rats/group): a control group fed on basal diet, four iron-supplemented groups fed on different iron levels $(105,175,350$ and $1400 \mathrm{mg}$ iron $/ \mathrm{kg}$ basal diet) and four iron + vitamin E-supplemented groups fed on the same different iron levels as well as $375 \mathrm{IU}$ of vitamin E/kg basal diet. Feeding iron-supplemented diets resulted in decrements of body weight gain, food intake and feed efficiency ratio, increases of relative organs weight, reductions of hemoglobin and hematocrit levels, liver function deterioration, iron and lipid profile disturbances, an increased lipid peroxidation level, a diminished antioxidant enzymes activity as well as histological alterations of liver tissues. Feeding iron + vitamin E-supplemented diets was found to ameliorate iron overload-induced hematological, biochemical and histological changes. Vitamin E supplementation may be useful for alleviating iron overload-induced side effects.
\end{abstract}

\section{Introduction}

Iron is an essential metal involved in a wide spectrum of physiological functions. It is an integral part of many proteins and enzymes. It is also essential for the regulation of cellular proliferation, differentiation and survival. Paradoxically, excess iron accumulation promotes the generation of free radicals, which lead to oxidative stress and oxidative tissue damage ${ }^{[1]}$.

The central importance of iron in the pathophysiology of disease is derived from the ease with which iron is reversibly oxidized and reduced. This property, while essential for its metabolic functions, makes iron potentially hazardous because of its ability to participate in the generation of powerful oxidant species which play a role in the generation of more reactive oxidants ${ }^{[2]}$.

\footnotetext{
* Corresponding author.

E-mail address: amromohsen321@yahoo.com
}

Iron can react with superoxide $\left(\mathrm{O}_{2}{ }^{-}\right)$and hydrogen peroxide $\left(\mathrm{H}_{2} \mathrm{O}_{2}\right)$, from activated neutrophils, to produce the hydroxyl radical $\left(\mathrm{OH}^{\circ}\right)$ via the Fenton reaction: 1. $\mathrm{O}_{2}^{-}+\mathrm{Fe}^{3+} \rightarrow \mathrm{O}_{2}+\mathrm{Fe}^{2+} ; 2 . \mathrm{H}_{2} \mathrm{O}_{2}+\mathrm{Fe}^{2+} \rightarrow \mathrm{Fe}^{3+}+\mathrm{OH}^{-}+$ $\mathrm{OH} \cdot$. Hydroxyl radicals are extremely reactive and can attack any cell components and cause oxidative damage. These radicals can also lead to the formation of other reactive oxygen species (ROS) ${ }^{[3]}$.

The health effects of iron excess have received increased attention as iron has been purported to play a critical role in the etiology of several chronic diseases, including diabetes, cardiovascular disease and cancer ${ }^{[4]}$.

Food fortification refers to the addition of micronutrients to processed foods. This strategy can lead to relatively rapid improvements in the micronutrients status of the population. Supplementation is the term used to describe the provision of relatively large doses of micronutrients, 
usually in the form of pills, capsules or syrups. It has the advantage of being capable of supplying an optimal amount of a specific nutrient or nutrients, in a highly absorbable form, and is often the fastest way to control deficiency in individuals and population groups that have been identified as being deficient ${ }^{[5]}$.

The popularity of dietary supplements to promote health and prevent disease has increased substantially in recent years. Vitamin $\mathrm{E}$ is one of the most common antioxidant supplements consumed. Vitamin E consists of a group of eight molecules belonging to two classes designed as tocopherols and tocotrienols. Both tocopherols and tocotrienols possess four structurally similar forms designated as alpha, beta, gamma and delta. In the past it was believed that each of these forms had a different degree of biological activity, with $\alpha$-tocopherol having the greatest. Vitamin E, a critical component of the antioxidant system in all tissues, is a free radical scavenger that has recently received attention as a potential therapeutic agent to prevent or reduce clinical disease states thought to be associated with excess free radical production. The health benefits of consuming vitamin $\mathrm{E}$ are believed to be attributed to its antioxidant properties ${ }^{[6]}$.

The impact of vitamin $\mathrm{E}$ on iron metabolism has been poorly investigated. Early studies have shown that experimentally-induced vitamin E deficiency is associated with increased susceptibility to iron toxicity. The contrasting roles of iron and vitamin $\mathrm{E}$ with respect to free radical metabolism suggest that an altered vitamin $\mathrm{E}$ status may play a role in the pathogenesis and treatment of iron toxicity ${ }^{[7]}$.

In an attempt to understand the role of vitamin $\mathrm{E}$ against iron toxicity, the present study was designed to evaluate the alleviating effect of vitamin E against iron-induced oxidative stress.

\section{Materials and methods \\ Experimental animals}

A total of seventy-two adult male albino rats of Sprague Dawley strain weighing 100-120 g were obtained from the National Nutrition Institute, Cairo, Egypt. All animal handling procedures were approved by the ethics committee of the National Nutrition Institute, Cairo, Egypt and in accordance with the recommendations for the proper care and use of laboratory animals (National Institutes of Health $[\mathrm{NIH}]$ publication No.85-23, revised 1996).

\section{Diet}

The basal diet was prepared according to the AIN-93 (American Institute of Nutrition) formulation as previously described ${ }^{[8]}$. Animals were allowed free access to the basal diet for 7 days as an adaptation period before starting the study. During the feeding period, food and water were provided ad-libitum. Rats were individually weighed at the start of the study. Body weight and food intake were recorded twice weekly to monitor body weight gain, feed intake and food efficiency ratio.

\section{Chemicals}

Ferrous sulphate $\left(\mathrm{FeSO}_{4} .7 \mathrm{H}_{2} \mathrm{O}\right)$ and $\alpha$-tocopherol acetate (vitamin E) were purchased from Sigma-Aldrich (St. Louis, MO, USA).

\section{Experimental design}

After the acclimation period, rats were divided into 9 groups (8 rats/group):

Group 1: Fed on basal diet for 8 weeks and served as a control group.

The other 8 groups were fed on iron overloaded diets with or without vitamin $\mathrm{E}$ for 8 weeks as follows:

Group 2: Fed on basal diet containing $105 \mathrm{mg}$ iron $/ \mathrm{kg}$ basal diet.

Group 3: Fed on basal diet containing $105 \mathrm{mg}$ iron +375 IU vitamin $\mathrm{E} / \mathrm{kg}$ basal diet.

Group 4: Fed on basal diet containing $175 \mathrm{mg}$ iron/ kg basal diet.

Group 5: Fed on basal diet containing $175 \mathrm{mg}$ iron +375 IU vitamin $\mathrm{E} / \mathrm{kg}$ basal diet.

Group 6: Fed on basal diet containing $350 \mathrm{mg}$ iron $/ \mathrm{kg}$ basal diet.

Group 7: Fed on basal diet containing $350 \mathrm{mg}$ iron +375

IU vitamin $\mathrm{E} / \mathrm{kg}$ basal diet.

Group 8: Fed on basal diet containing $1400 \mathrm{mg}$ iron $/ \mathrm{kg}$ basal diet.

Group 9: Fed on basal diet containing $1400 \mathrm{mg}$ iron + 375 IU vitamin E/kg basal diet ${ }^{[9]}$.

At the end of the experimental period of 8 weeks, rats were kept fasting overnight and then anaesthetized by inhalation of diethyl ether. Blood samples were withdrawn from the hepatic portal vein on heparin. Erythrocytes and plasma samples were separated and stored at $-80^{\circ} \mathrm{C}$ until analysis. The liver, heart, spleen and kidneys from dissected rats was immediately excised, blood was cleared off by several washings with ice cold phosphate buffered saline and the tissues were weighed. Portions of the liver tissues were sectioned and kept in $10 \%$ formalin for histological analysis.

\section{Biological studies}

\section{Body weight gain}

At the beginning of the study, all rats were individually weighed twice weekly in the first 4 weeks, then once weekly in the second 4 weeks. Body weight gain was calculated according to the following equation as previously described ${ }^{[9]}$ : Body weight gain $(\mathrm{gm})=$ Final weight - Initial weight.

\section{Food intake}

The total diet consumed per group during the experimental period was calculated as previously documented ${ }^{[10]}$. The amount of food consumed per group was calculated by subtracting the amount of food waste from the total amount of food allocated to the rats in each group. Mean food consumption was calculated by dividing the amount of food consumed per group by the number of rats in the respective group. Food intake (gm/day) was calculated by dividing the mean food consumption per group by the experimental period. 


\section{Feed efficiency ratio}

Feed efficiency ratio was calculated according to the following equation: Feed efficiency ratio $=$ Body weight gain $(\mathrm{gm})$ of rats in each group / food intake (gm) of rats in each group ${ }^{[10]}$.

\section{Relative organs weight}

Relative weights of the liver, heart, kidneys and spleen were calculated as previously described ${ }^{[10]}$. Relative weights $=$ (Absolute weight of organ / Total weight of rat at the end of the experiment $) \times 100$.

\section{Biochemical investigations}

\section{Hemoglobin}

Hemoglobin concentration was determined according to a previously described method ${ }^{[11]}$, using a commercially available kit (BioMed Diagnostics, Egy-Chem, Egypt).

\section{Hematocrit}

Hematocrit, also known as packed cell volume, was determined according to a previously described method [12]

\section{Iron and total iron binding capacity}

Plasma iron and total iron binding capacity (TIBC) were determined according to a previously described method $[13,14]$

\section{Transferrin saturation}

Transferrin saturation was calculated as according to the previously described formula: Transferrin saturation $=$ (Plasma iron / total iron binding capacity) $\times 100^{[15]}$.

\section{Ferritin}

Plasma ferritin concentration was determined according to a previously described method ${ }^{[16]}$, using enzyme linked immunosorbent assay (ELISA; Microwell Ferritin EIA, Syntron Bioresearch Inc. Carlsbad, CA, USA).

\section{Liver function tests}

Plasma alanine aminotransferase (ALT) and aspartate aminotransferase (AST) were assessed in units as previously described ${ }^{[17]}$, using commercially available kits (BioMed Diagnostics, Egy-Chem, Egypt). Plasma alkaline phosphatase (ALP) was assessed as previously described [18], using a commercially available kit (BioMed Diagnostics, Egy-Chem, Egypt).(one unit of ALT is defined as : The amount of enzyme that generates $1.0 \mu$ mole of pyruvate per minute at $37^{\circ} \mathrm{C}$, one unit of AST is defined as : The amount of enzyme that generates $1.0 \mu$ mole of glutamate per minute at $\mathrm{pH}$ 8.0 at $\left.37^{\circ} \mathrm{C}\right)$.

\section{Lipid profile}

Plasma total cholesterol (TC) concentration was determined as previously described [19], using a commercially available kit (BioMed Diagnostics, EgyChem, Egypt). Plasma triglycerides (TG) concentration was determined as previously described ${ }^{[20]}$, using a commercially available kit (BioMed Diagnostics, EgyChem, Egypt). Plasma high-density lipoprotein cholesterol (HDL-C) concentration was determined as previously described ${ }^{[21]}$, using a commercially available kit (BioMed Diagnostics, Egy-Chem, Egypt). Plasma low-density lipoprotein cholesterol (LDL-C) and very low density lipoprotein cholesterol (VLDL-C) levels were calculated using a previously described formula ${ }^{[22]}$. $\mathrm{C}_{\mathrm{LDL}}=\mathrm{C}_{\text {Cholest. }}-\mathrm{C}_{\mathrm{HDL}}-\mathrm{TG} / 5$

$\mathrm{VLDL}=\mathrm{TG} / 5$

\section{Lipid peroxidation level}

Plasma malondialdehyde (MDA) level was determined according to a previously described method ${ }^{[23]}$.

\section{Antioxidant enzymes activity}

Erythrocytes superoxide dismutase (SOD) activity was determined in units according to a previously described method [24]. Plasma catalase (CAT) activity was determined in units according to a previously described method ${ }^{[25]}$. One SOD unit definition: one unit will inhibit the rate of reduction of cytochrome $\mathrm{C}$ by $50 \%$ in a coupled system, using Xanthine oxidase at $\mathrm{pH} 7.8$ at $25^{\circ} \mathrm{C}$, Catalase unit definition: One unit of catalase will decompose $1.0 \mu$ mole of $\mathrm{H}_{2} \mathrm{O}_{2}$ per minute at $\mathrm{pH} 7$ and $25^{\circ} \mathrm{C}$.

\section{Total antioxidant capacity}

Total antioxidant capacity (TAC) was determined in the plasma according to a previously described method ${ }^{[26]}$, using a commercially available kit (Biodiagnostics Inc., Egypt).

\section{Vitamin E}

Plasma vitamin E ( $\alpha$-tocopherol) level was determined as previously described ${ }^{[27]}$, using high performance liquid chromatography (HPLC).

\section{Histological studies}

Portions of the liver tissues were fixed in $10 \%$ neutralbuffered formalin, dehydrated in graded alcohol series, cleared in xylene, embedded in paraffin, sliced into 5- $\mu \mathrm{m}$ sections, stained with hematoxylin-eosin (H\&E), followed by histological assessment as previously described ${ }^{[28]}$.

\section{Statistical analysis}

Data were expressed as mean \pm standard deviation (SD). The statistical significance of differences between means was analyzed using one-way analysis of variance (ANOVA). Statistical significance was set at a $P$ value of $<0.05$. Data statistical analyses were performed using the statistical package for the social sciences (SPSS Statistics for Windows, Version 20.0; IBM Corp., Armonk, NY, USA).

\section{Results}

\section{Biological studies}

As shown in Tables $\mathbf{1}$ and 2, feeding iron-supplemented diets resulted in decrements of body weight gain, food intake and feed efficiency ratio, with concomitant increases of relative weight of liver, heart, kidneys and spleen, as compared to the control group. On the other hand, feeding iron + vitamin E-supplemented diets was found to ameliorate iron overload-induced changes in body weight gain, food intake, feed efficiency ratio and relative organs weight, in comparison to iron-overloaded groups. 
Table 1: Body weight gain, food intake and feed efficiency ratio of the study groups

\begin{tabular}{|c|c|c|c|c|c|c|c|c|c|}
\hline Parameters & $\begin{array}{c}\text { Control } \\
\text { (1) }\end{array}$ & $\begin{array}{c}105 \mathrm{mg} \mathrm{Fe} \\
(2)\end{array}$ & $\begin{array}{c}105 \mathrm{mg} \mathrm{Fe} \\
+ \text { Vitamin } \mathrm{E} \\
\text { (3) }\end{array}$ & $\begin{array}{c}175 \mathrm{mg} \mathrm{Fe} \\
\text { (4) }\end{array}$ & $\begin{array}{c}175 \mathrm{mg} \mathrm{Fe} \\
+ \text { Vitamin } \mathrm{E} \\
(5)\end{array}$ & $\begin{array}{c}350 \mathrm{mg} \mathrm{Fe} \\
(6)\end{array}$ & $\begin{array}{c}350 \mathrm{mg} \text { Fe } \\
+ \text { Vitamin } \mathrm{E} \\
(7)\end{array}$ & $\begin{array}{c}1400 \mathrm{mg} \mathrm{Fe} \\
(8)\end{array}$ & $\begin{array}{l}1400 \mathrm{mg} \mathrm{Fe} \\
+ \text { Vitamin E } \\
(9)\end{array}$ \\
\hline $\begin{array}{l}\text { Body weight } \\
\text { gain }(\mathrm{g})\end{array}$ & $82.56 \pm 6.65$ & $78.25 \pm 9.57$ & $81.5 \pm 9.05$ & $42.5 \pm 11.77$ & $65.25 \pm 6.4$ & $24.5 \pm 2.67$ & $50.6 \pm 9.37$ & $6.5 \pm 5.4$ & $24 \pm 2.92$ \\
\hline \multirow{2}{*}{$\%$ change } & & $-5.2 \% \mathrm{c}$ & $-1.3 \% \mathrm{c}$ & $-48.52 \% \mathrm{c}$ & $-20.66 \% \mathrm{c}$ & $-70.32 \% \mathrm{c}$ & $-38.71 \% \mathrm{c}$ & $-92.1 \% \mathrm{c}$ & $-70.9 \% \mathrm{c}$ \\
\hline & & & $4.15 \% \mathrm{~d}$ & & $53.5 \% \mathrm{~d}$ & & $106.5 \% \mathrm{~d}$ & & $269.2 \% \mathrm{~d}$ \\
\hline Significance & & N.S & N.S & $a^{* *}$ & $a * b^{* *}$ & $a^{* *}$ & $a^{* *} b^{* *}$ & $a^{* *}$ & $a^{* *} b^{* *}$ \\
\hline $\begin{array}{l}\text { Food intake } \\
\text { (g/days) }\end{array}$ & $9.14 \pm 0.92$ & $8.97 \pm 0.76$ & $9.22 \pm 0.76$ & $8.37 \pm 0.82$ & $8.78 \pm 1.04$ & $7.32 \pm 0.90$ & $8.76 \pm 1.21$ & $7.16 \pm 0.54$ & $7.53 \pm 0.94$ \\
\hline \multirow{2}{*}{$\%$ change } & & $-1.86 \% \mathrm{c}$ & $0.87 \% \mathrm{c}$ & $-8.42 \% \mathrm{c}$ & $-3.94 \% \mathrm{c}$ & $-19.91 \% \mathrm{c}$ & $-4.15 \% \mathrm{c}$ & $-21.66 \% \mathrm{c}$ & $-17.6 \% \mathrm{c}$ \\
\hline & & & $2.8 \% \mathrm{~d}$ & & $4.9 \% \mathrm{~d}$ & & $19.67 \% \mathrm{~d}$ & & $5.16 \% \mathrm{~d}$ \\
\hline Significance & & N.S & N.S & $a^{*}$ & N.Sb* & $\mathrm{a}^{*}$ & N.Sb* & $\mathrm{a}^{* *}$ & $a^{* *} b^{*}$ \\
\hline $\begin{array}{l}\text { Feed efficiency } \\
\text { ratio }\end{array}$ & $0.16 \pm 0.04$ & $0.15 \pm 0.04$ & $0.16 \pm 0.05$ & $0.09 \pm 0.05$ & $0.13 \pm 0.04$ & $0.06 \pm 0.04$ & $0.09 \pm 0.07$ & $0.01 \pm 0.02$ & $0.05 \pm 0.03$ \\
\hline \multirow{2}{*}{$\%$ change } & & $-6.6 \% \mathrm{c}$ & 0 & $-43.75 \% \mathrm{c}$ & $-18.75 \% \mathrm{c}$ & $-62.5 \% \mathrm{c}$ & $-43.75 \% \mathrm{c}$ & $-93.75 \% \mathrm{c}$ & $-68.75 \% c$ \\
\hline & & & $6.6 \% \mathrm{~d}$ & & $44.4 \% \mathrm{~d}$ & & $50 \% \mathrm{~d}$ & & $400 \% \mathrm{~d}$ \\
\hline Significance & & N.S & N.S & $a^{*}$ & $a * b *$ & $a^{* *}$ & $a * b *$ & $a^{* *}$ & $a^{* *} b^{* *}$ \\
\hline
\end{tabular}

Data are represented as mean \pm S.D (8 rats / group).

a: indicates the statistical difference in comparison to the control group.

b: indicates the statistical difference between the four iron-supplemented groups $(2,4,6$ and 8$)$ and the four iron + vitamin E-supplemented groups (3, 5, 7 and 9).

c: \% change compared to the control group.

$\mathrm{d}$ : \% change of the four iron-supplemented groups (2, 4, 6 and 8) compared to the four iron + vitamin E-supplemented groups (3, 5, 7 and 9)

**: $\mathrm{p}<0.01$ : highly significant. *: $\mathrm{p}<0.05$ : significant. N.S: Non-significant.

Table 2: Relative organs weight of the study groups

\begin{tabular}{|c|c|c|c|c|c|c|c|c|c|}
\hline Parameters & $\begin{array}{l}\text { Control } \\
\text { (1) }\end{array}$ & $\begin{array}{c}105 \mathrm{mg} \mathrm{Fe} \\
\text { (2) }\end{array}$ & $\begin{array}{c}105 \mathrm{mg} \mathrm{Fe} \\
+ \text { Vitamin } \mathrm{E} \\
\text { (3) }\end{array}$ & $\begin{array}{c}175 \mathrm{mg} \mathrm{Fe} \\
\text { (4) }\end{array}$ & $\begin{array}{c}175 \mathrm{mg} \mathrm{Fe} \\
+ \text { Vitamin E } \\
(5)\end{array}$ & $\begin{array}{c}350 \mathrm{mg} \mathrm{Fe} \\
\text { (6) }\end{array}$ & $\begin{array}{c}350 \mathrm{mg} \mathrm{Fe} \\
+ \text { Vitamin } \mathrm{E} \\
(7)\end{array}$ & $\begin{array}{c}1400 \mathrm{mg} \mathrm{Fe} \\
\text { (8) }\end{array}$ & $\begin{array}{c}1400 \mathrm{mg} \mathrm{Fe} \\
+ \text { Vitamin E } \\
(9)\end{array}$ \\
\hline $\begin{array}{l}\text { Liver }(\mathrm{g} / 100 \mathrm{~g} \\
\text { body weight) }\end{array}$ & $5.1 \pm 0.36$ & $5.24 \pm 0.19$ & $5.15 \pm 0.23$ & $5.51 \pm 0.13$ & $5.31 \pm 0.41$ & $6.08 \pm 0.29$ & $5.68 \pm 0.23$ & $6.70 \pm 0.76$ & $6.20 \pm 0.59$ \\
\hline \multirow{2}{*}{$\%$ change } & & $2.74 \% \mathrm{c}$ & $1 \% \mathrm{c}$ & $8 \% \mathrm{c}$ & $4.12 \% \mathrm{c}$ & $19.21 \% \mathrm{c}$ & $11.37 \% \mathrm{c}$ & $31.37 \% \mathrm{c}$ & $21.56 \% \mathrm{c}$ \\
\hline & & & $-1.7 \% \mathrm{~d}$ & & $-3.63 \% d$ & & $-6.58 \% \mathrm{~d}$ & & $-7.46 \% \mathrm{~d}$ \\
\hline Significance & & N.S & N.S & $\mathrm{a}^{*}$ & N.S & $\mathrm{a}^{* *}$ & $\mathrm{a}^{* *}$ & $\mathrm{a}^{* *}$ & $a^{* *}$ \\
\hline $\begin{array}{l}\text { Heart }(\mathrm{g} / 100 \mathrm{~g} \\
\text { body weight) }\end{array}$ & $0.32 \pm 0.03$ & $0.36 \pm 0.05$ & $0.35 \pm 0.07$ & $0.38 \pm 0.05$ & $0.37 \pm 0.06$ & $0.41 \pm 0.05$ & $0.4 \pm 0.03$ & $0.43 \pm 0.09$ & $0.42 \pm 0.05$ \\
\hline \multirow{2}{*}{$\%$ change } & & $12.5 \% \mathrm{c}$ & $9.37 \% \mathrm{c}$ & $18.75 \% \mathrm{c}$ & $15.62 \% \mathrm{c}$ & $28.12 \% \mathrm{c}$ & $25 \% \mathrm{c}$ & $34.37 \% \mathrm{c}$ & $31.25 \% \mathrm{c}$ \\
\hline & & & $-2.78 \% \mathrm{~d}$ & & $-2.63 \% \mathrm{~d}$ & & $-2.44 \% \mathrm{~d}$ & & $-2.32 \% \mathrm{~d}$ \\
\hline Significance & & N.S & N.S & $a^{*}$ & N.S & $a^{* *}$ & $a^{* *}$ & $a^{* *}$ & $a^{* * *}$ \\
\hline $\begin{array}{l}\text { Kidneys (g/100g } \\
\text { body weight) }\end{array}$ & $1.3 \pm 0.02$ & $1.82 \pm 0.25$ & $1.33 \pm 0.1$ & $1.9 \pm 0.4$ & $1.48 \pm 0.58$ & $2.0 \pm 0.3$ & $1.48 \pm 0.26$ & $2.10 \pm 0.27$ & $1.7 \pm 0.20$ \\
\hline \multirow{2}{*}{$\%$ change } & & $40 \% \mathrm{c}$ & $2.3 \% \mathrm{c}$ & $46.15 \% \mathrm{c}$ & $13.84 \% \mathrm{c}$ & $53.84 \%$ & $13.84 \% \mathrm{c}$ & $61.54 \% \mathrm{c}$ & $30.77 \% \mathrm{c}$ \\
\hline & & & $-26.92 \% \mathrm{~d}$ & & $-22.1 \% \mathrm{~d}$ & & $-26 \% \mathrm{~d}$ & & $-19 \% \mathrm{~d}$ \\
\hline Significance & & $a^{* *}$ & N.S b** & $a^{* *}$ & N.S b* & $a^{* *}$ & N.S b* & $a^{* *}$ & $a^{* *} b^{*}$ \\
\hline $\begin{array}{l}\text { Spleen }(\mathrm{g} / 100 \mathrm{~g} \\
\text { body weight) }\end{array}$ & $0.46 \pm 0.11$ & $0.54 \pm 0.04$ & $0.50 \pm 0.02$ & $0.54 \pm 0.05$ & $0.52 \pm 0.03$ & $0.58 \pm 0.06$ & $0.53 \pm 0.10$ & $0.64 \pm 0.02$ & $0.60 \pm 0.07$ \\
\hline \multirow{2}{*}{$\%$ change } & & $17.4 \% \mathrm{c}$ & $8.7 \% \mathrm{c}$ & $17.4 \% \mathrm{c}$ & $13.04 \% \mathrm{c}$ & $26.1 \% \mathrm{c}$ & $15.22 \% \mathrm{c}$ & $39.13 \% \mathrm{c}$ & $30.43 \% \mathrm{c}$ \\
\hline & & & $-7.4 \% \mathrm{~d}$ & & $-3.7 \% \mathrm{~d}$ & & $-8.6 \% \mathrm{~d}$ & & $-6.25 \% \mathrm{~d}$ \\
\hline Significance & & $a^{*}$ & N.S & $a^{*}$ & N.S & $a^{*}$ & N.S & $a^{* *}$ & $a^{*}$ \\
\hline
\end{tabular}

Data are represented as mean \pm S.D (8 rats / group).

a: indicates the statistical difference in comparison to the control group.

b: indicates the statistical difference between the four iron-supplemented groups $(2,4,6$ and 8$)$ and the four iron + vitamin E-supplemented groups $(3,5,7$ and 9$)$.

c: \% change compared to the control group.

$\mathrm{d}$ : \% change of the four iron-supplemented groups (2, 4, 6 and 8) compared to the four iron + vitamin E-supplemented groups (3, 5, 7 and 9)

$* *$ : $<<0.01$ : highly significant. *: $\mathrm{p}<0.05$ : significant. N.S: Non-significant. 


\section{Hematological studies}

Table 3 shows that feeding iron-supplemented diets resulted in reductions of hemoglobin and hematocrit levels, when compared to the control group. On the other hand, feeding iron + vitamin E-supplemented diets was found to cause a remarkable elevation of hemoglobin and hematocrit levels, in comparison to iron-overloaded groups.

\section{Biochemical studies}

\section{Iron profile}

As summarized in Table 3, feeding iron-supplemented diets resulted in significant elevations of plasma levels of iron, ferritin and transferrin saturation, with concomitant depletions of total iron binding capacity, as compared to the control group. On the other hand, feeding iron +vitamin E-supplemented diets resulted in a significant decrease of plasma levels of iron, ferritin and transferrin saturation, while increasing total iron binding capacity, in comparison to iron-overloaded groups.

\section{Liver function tests}

As illustrated in Table 4, feeding iron-supplemented diets resulted in increased plasma activities of ALT, AST and ALP, when compared to the control group. On the other hand, feeding iron + vitamin E-supplemented diets resulted in a remarkable decrease of plasma activities of ALT, AST and ALP, in comparison to iron-overloaded groups.

\section{Lipid profile}

Table 5 shows that feeding iron-supplemented diets resulted in significant elevations of plasma levels of TC, TG, LDL-C and VLDL-C, with concomitant depletions of HDL-C plasma levels, when compared to the control group. On the other hand, feeding iron + vitamin Esupplemented diets resulted in significant decreases of plasma levels of TC, TG, LDL-C and VLDL-C, while significantly increasing HDL-C plasma levels, in comparison to iron-overloaded groups.

Table 3: Hemoglobin and hematocrit levels and plasma iron profile of the study groups

\begin{tabular}{|c|c|c|c|c|c|c|c|c|c|}
\hline Parameters & $\begin{array}{l}\text { Control } \\
\text { (1) }\end{array}$ & $\begin{array}{c}105 \mathrm{mg} \mathrm{Fe} \\
(2) \\
\end{array}$ & $\begin{array}{c}105 \mathrm{mg} \mathrm{Fe} \\
+ \text { Vitamin E } \\
(3)\end{array}$ & $\begin{array}{c}175 \mathrm{mg} \mathrm{Fe} \\
(4)\end{array}$ & $\begin{array}{c}175 \mathrm{mg} \mathrm{Fe} \\
+ \text { Vitamin } \mathrm{E} \\
(5)\end{array}$ & $\begin{array}{c}350 \mathrm{mg} \mathrm{Fe} \\
(6)\end{array}$ & $\begin{array}{c}350 \mathrm{mg} \mathrm{Fe} \\
+ \text { Vitamin } \mathrm{E} \\
(7)\end{array}$ & $\begin{array}{c}1400 \mathrm{mg} \mathrm{Fe} \\
(8)\end{array}$ & $\begin{array}{c}1400 \mathrm{mg} \mathrm{Fe} \\
+ \text { Vitamin E } \\
(9)\end{array}$ \\
\hline Hemoglobin $(\mathrm{g} / \mathrm{dl})$ & $13.87 \pm 0.27$ & $12.51 \pm 0.35$ & $13.66 \pm 0.27$ & $12.0 \pm 0.63$ & $13.42 \pm 0.38$ & $11.6 \pm 0.32$ & $13.3 \pm 0.36$ & $10.8 \pm 0.55$ & $12.7 \pm 0.68$ \\
\hline \multirow{2}{*}{$\%$ change } & & $-9.8 \% c$ & $-1.51 \% \mathrm{c}$ & $-13.5 \% \mathrm{c}$ & $-3.24 \% \mathrm{c}$ & $-16.36 \% \mathrm{c}$ & $-4.10 \% \mathrm{c}$ & $-22.13 \% c$ & $-8.43 \% \mathrm{c}$ \\
\hline & & & $9.2 \% \mathrm{~d}$ & & $11.83 \% \mathrm{~d}$ & & $14.65 \% \mathrm{~d}$ & & $17.6 \% \mathrm{~d}$ \\
\hline Significance & & $a^{* *}$ & N.S b** & $a^{* *}$ & N.S b** & $a^{* *}$ & N.S b** & $a^{* *}$ & $\mathrm{a}^{*} \mathrm{~b}^{* *}$ \\
\hline Hematocrit & $42.54 \pm 2.15$ & $38.41 \pm 1.3$ & $41.98 \pm 0.83$ & $37.0 \pm 1.91$ & $41.27 \pm 1.14$ & $35.8 \pm 0.96$ & $41.12 \pm 1.1$ & $33.47 \pm 1.66$ & $39.1 \pm 2.04$ \\
\hline \multirow{2}{*}{$\%$ change } & & $-9.7 \% \mathrm{c}$ & $-1.31 \% \mathrm{c}$ & $-13.0 \% \mathrm{c}$ & $-3 \% c$ & $-15.84 \% \mathrm{c}$ & $-3.33 \% \mathrm{c}$ & $-21.32 \% c$ & $-8.1 \% \mathrm{c}$ \\
\hline & & & $9.3 \% \mathrm{~d}$ & & $11.54 \% \mathrm{~d}$ & & $14.86 \% \mathrm{~d}$ & & $16.82 \% \mathrm{~d}$ \\
\hline Significance & & $a^{* *}$ & N.S b** & $\mathrm{a}^{* *}$ & N.S b** & $a^{* * *}$ & N.S b** & $a^{* *}$ & $\mathrm{a}^{*} \mathrm{~b}^{* *}$ \\
\hline Iron $(\mu \mathrm{g} / \mathrm{dl})$ & $250.85 \pm 4.4$ & $268.43 \pm 4.65$ & $252.45 \pm 5.22$ & $298.1 \pm 2.32$ & $264.45 \pm 4.64$ & $329.46 \pm 4.32$ & $273.65 \pm 6.18$ & $382.9 \pm 5.33$ & $283.5 \pm 3.86$ \\
\hline \multirow{2}{*}{$\%$ change } & & $7 \% \mathrm{c}$ & $0.63 \% \mathrm{c}$ & $18.83 \% \mathrm{c}$ & $5.42 \% \mathrm{c}$ & $31.33 \% \mathrm{c}$ & $9.1 \% \mathrm{c}$ & $52.64 \% \mathrm{c}$ & $13 \% \mathrm{c}$ \\
\hline & & & $(-) 5.95 \% \mathrm{~d}$ & & $(-) 11.3 \% \mathrm{~d}$ & & $(-) 16.9 \% \mathrm{~d}$ & & $(-) 25.96 \% \mathrm{~d}$ \\
\hline Significance & & $a^{* * *}$ & N.S b** & $a^{* *}$ & $\mathrm{a}^{* *} \mathrm{~b} * *$ & $a^{* *}$ & $a * * b * *$ & $a^{* *}$ & $\mathrm{a}^{* * \mathrm{~b} * *}$ \\
\hline TIBC $(\mu \mathrm{g} / \mathrm{dl})$ & $671.53 \pm 2.23$ & $662.58 \pm 3.05$ & $783.80 \pm 3.65$ & $643.64 \pm 3.17$ & $805 \pm 3.97$ & $613.81 \pm 4.39$ & $824.53 \pm 2.4$ & $587.24 \pm 3.93$ & $842.1 \pm 3.35$ \\
\hline \multirow{2}{*}{$\%$ change } & & $-1.33 \% \mathrm{c}$ & $16.7 \% \mathrm{c}$ & $-4.15 \% \mathrm{c}$ & $19.87 \% \mathrm{c}$ & $-8.6 \% \mathrm{c}$ & $22.78 \% \mathrm{c}$ & $-12.55 \% \mathrm{c}$ & $25.4 \% \mathrm{c}$ \\
\hline & & & $18.3 \% \mathrm{~d}$ & & $25.11 \% \mathrm{~d}$ & & $34.33 \% \mathrm{~d}$ & & $43.4 \% \mathrm{~d}$ \\
\hline Significance & & N.S & $\mathrm{a}^{* *} \mathrm{~b}^{* *}$ & $a^{* *}$ & $\mathrm{a}^{* *} \mathrm{~b} * *$ & $\mathrm{a}^{* * *}$ & $a * * b * *$ & $a^{* *}$ & $\mathrm{a}^{* * \mathrm{~b}^{* *}}$ \\
\hline Ferritin (ng/ml) & $148.25 \pm 7.61$ & $178.86 \pm 2.77$ & $158.44 \pm 4.35$ & $189.77 \pm 3.75$ & $166.30 \pm 5.04$ & $200.91 \pm 5.03$ & $182.76 \pm 3.81$ & $249.03 \pm 3.61$ & $203.80 \pm 4.96$ \\
\hline \multirow{2}{*}{$\%$ change } & & $20.64 \% \mathrm{c}$ & $6.87 \% \mathrm{c}$ & $28 \% \mathrm{c}$ & $12.2 \% \mathrm{c}$ & $35.52 \% \mathrm{c}$ & $23.27 \% \mathrm{c}$ & $67.9 \% \mathrm{c}$ & $37.47 \% \mathrm{c}$ \\
\hline & & & $-11.41 \% \mathrm{~d}$ & & $-12.36 \% \mathrm{~d}$ & & $-9.03 \% \mathrm{~d}$ & & $-18.16 \% \mathrm{~d}$ \\
\hline Significance & & $\mathrm{a}^{* *}$ & $\mathrm{a}^{*} \mathrm{~b}^{* *}$ & $\mathrm{a}^{* *}$ & $\mathrm{a}^{* *} \mathrm{~b}^{* *}$ & $a^{* * *}$ & $\mathrm{a}^{* * \mathrm{~b}^{* *}}$ & $a^{* * *}$ & $\mathrm{a}^{* * \mathrm{~b}^{* *}}$ \\
\hline $\begin{array}{l}\text { (\%) Transferrin } \\
\text { saturation }\end{array}$ & $37.35 \pm 0.7$ & $40.51 \pm 0.72$ & $32.21 \pm 0.63$ & $46.60 \pm 1.63$ & $32.84 \pm 0.54$ & $53.67 \pm 0.8$ & $33.2 \pm 0.75$ & $65.21 \pm 1.15$ & $33.66 \pm 0.44$ \\
\hline \multirow{2}{*}{$\%$ change } & & $8.46 \% \mathrm{c}$ & $-13.76 \% c$ & $24.76 \% \mathrm{c}$ & $-12.1 \% \mathrm{c}$ & $43.7 \% \mathrm{c}$ & $-11.11 \% \mathrm{c}$ & $74.6 \% \mathrm{c}$ & $-9.9 \% \mathrm{c}$ \\
\hline & & & $-20.45 \% \mathrm{~d}$ & & $-29.52 \% \mathrm{~d}$ & & $-38.14 \% \mathrm{~d}$ & & $-48.38 \% \mathrm{~d}$ \\
\hline Significance & & $a^{* *}$ & $\mathrm{a}^{* *} \mathrm{~b}^{* *}$ & $a^{* *}$ & $a * * b * *$ & $a^{* * *}$ & $a * * b * *$ & $a^{* *}$ & $a^{* *} b^{* *}$ \\
\hline
\end{tabular}

Data are represented as mean \pm S.D (8 rats / group).

a: indicates the statistical difference in comparison to the control group.

b: indicates the statistical difference between the four iron-supplemented groups $(2,4,6$ and 8$)$ and the four iron + vitamin E-supplemented groups (3, 5, 7 and 9).

c: \% change compared to the control group.

d: \% change of the four iron-supplemented groups (2, 4, 6 and 8) compared to the four iron + vitamin E-supplemented groups (3, 5, 7 and 9)

$* *$ : $<<0.01$ : highly significant. *: p<0.05: significant. N.S: Non-significant. 
Table 4: Plasma ALT, AST and ALP levels of the study groups

\begin{tabular}{|c|c|c|c|c|c|c|c|c|c|}
\hline Parameters & $\begin{array}{l}\text { Control } \\
\text { (1) }\end{array}$ & $\begin{array}{c}105 \mathrm{mg} \mathrm{Fe} \\
\text { (2) }\end{array}$ & $\begin{array}{c}105 \mathrm{mg} \mathrm{Fe} \\
+ \text { Vitamin } \mathrm{E} \\
\text { (3) }\end{array}$ & $\begin{array}{c}175 \mathrm{mg} \mathrm{Fe} \\
\text { (4) }\end{array}$ & $\begin{array}{c}175 \mathrm{mg} \mathrm{Fe} \\
+ \text { Vitamin } \mathrm{E} \\
(\mathbf{5})\end{array}$ & $\begin{array}{c}350 \mathrm{mg} \mathrm{Fe} \\
(6)\end{array}$ & $\begin{array}{c}350 \mathrm{mg} \mathrm{Fe} \\
+ \text { Vitamin } \mathrm{E} \\
\quad(7)\end{array}$ & $\begin{array}{c}1400 \mathrm{mg} \mathrm{Fe} \\
(8)\end{array}$ & $\begin{array}{l}1400 \mathrm{mg} \mathrm{Fe} \\
+ \text { Vitamin } \mathrm{E} \\
\text { (9) }\end{array}$ \\
\hline ALT(U/L) & $.48 \pm 1.54$ & $26 \pm 1.15$ & $22.82 \pm 3.38$ & $27.92 \pm 7.16$ & $23.41 \pm 3.6$ & $33.03 \pm 5.5$ & $24.55 \pm 4.7$ & $39.73 \pm 3.95$ & $24.74 \pm 4.14$ \\
\hline \multirow{2}{*}{$\%$ change } & & $15.66 \% \mathrm{c}$ & $1.51 \% \mathrm{c}$ & $24.2 \% \mathrm{c}$ & $4.14 \% \mathrm{c}$ & $46.93 \% \mathrm{c}$ & $9.21 \% \mathrm{c}$ & $76.73 \% \mathrm{c}$ & $10.05 \% \mathrm{c}$ \\
\hline & & & $-12.2 \% \mathrm{~d}$ & & $-16.15 \% \mathrm{~d}$ & & $-25.67 \% \mathrm{~d}$ & & $-37.7 \% \mathrm{~d}$ \\
\hline Significance & & N.S & N.S b* & N.S & N.S b* & $a^{* *}$ & $\mathrm{NS} \mathrm{b}^{* * *}$ & $a^{* *}$ & N.S b** \\
\hline $\operatorname{AST}(\mathrm{U} / \mathrm{L})$ & $28.08 \pm 3.18$ & $33.5 \pm 5.96$ & $29.13 \pm 4.22$ & $38.61 \pm 4.47$ & $30.3 \pm 4.58$ & $43.61 \pm 5.17$ & $32.23 \pm 6.73$ & $54.78 \pm 5.97$ & $32.76 \pm 6.07$ \\
\hline \multirow{2}{*}{$\%$ change } & & $19.3 \% \mathrm{c}$ & $3.74 \% \mathrm{c}$ & $37.5 \% \mathrm{c}$ & $7.9 \% \mathrm{c}$ & $55.3 \% \mathrm{c}$ & $14.78 \% \mathrm{c}$ & $95.08 \% \mathrm{c}$ & $16.66 \% \mathrm{c}$ \\
\hline & & & $-13 \% d$ & & $-21.5 \% \mathrm{~d}$ & & $-26.1 \% d$ & & $-40.2 \% \mathrm{~d}$ \\
\hline Significance & & $a^{*}$ & N.S b* & $a^{* * *}$ & N.S b** & $a^{* *}$ & N.S b** & $a^{* *}$ & $a^{*} b^{* *}$ \\
\hline ALP (U/L) & $38.94 \pm 1.78$ & $42.36 \pm 2.36$ & $39.16 \pm 2.17$ & $45.14 \pm 2.12$ & $41.44 \pm 1.8$ & $49.63 \pm 2.66$ & $42.63 \pm 2.05$ & $55.15 \pm 3.04$ & $44.24 \pm 2$ \\
\hline \multirow{2}{*}{$\% \overline{\text { change }}$} & & $8.78 \% \mathrm{c}$ & $0.56 \% \mathrm{c}$ & $15.92 \% \mathrm{c}$ & $6.42 \% \mathrm{c}$ & $27.45 \% \mathrm{c}$ & $9.47 \% \mathrm{c}$ & $41.62 \% \mathrm{c}$ & $13.61 \% \mathrm{c}$ \\
\hline & & & $-7.55 \% \mathrm{~d}$ & & $-8.2 \% \mathrm{~d}$ & & $-14.1 \% \mathrm{~d}$ & & $-19.8 \% \mathrm{~d}$ \\
\hline Significance & & $a^{*}$ & N.S b* & $a^{* * *}$ & $a * b * *$ & $a^{* * *}$ & $\mathrm{a}^{*} \mathrm{~b}^{* * *}$ & $a^{* * *}$ & $a^{* *} b^{* *}$ \\
\hline
\end{tabular}

Data are represented as mean \pm S.D (8 rats / group).

a: indicates the statistical difference in comparison to the control group.

b: indicates the statistical difference between the four iron-supplemented groups $(2,4,6$ and 8$)$ and the four iron + vitamin E-supplemented groups (3, 5, 7 and 9).

c: \% change compared to the control group.

$\mathrm{d}$ : \% change of the four iron-supplemented groups (2, 4, 6 and 8) compared to the four iron + vitamin E-supplemented groups (3, 5, 7 and 9)

**: $\mathrm{p}<0.01$ : highly significant. *: $\mathrm{p}<0.05$ : significant. N.S: Non-significant.

Table 5: Plasma lipid profile of the study groups

\begin{tabular}{|c|c|c|c|c|c|c|c|c|c|}
\hline Parameters & $\begin{array}{l}\text { Control } \\
\text { (1) }\end{array}$ & $\begin{array}{c}105 \mathrm{mg} \text { Fe } \\
(2)\end{array}$ & $\begin{array}{c}105 \mathrm{mg} \mathrm{Fe} \\
+ \text { Vitamin } \mathrm{E} \\
(3)\end{array}$ & $\begin{array}{c}175 \mathrm{mg} \mathrm{Fe} \\
(4)\end{array}$ & $\begin{array}{c}175 \mathrm{mg} \mathrm{Fe} \\
+ \text { Vitamin E } \\
(5)\end{array}$ & $\begin{array}{c}350 \mathrm{mg} \mathrm{Fe} \\
(6)\end{array}$ & $\begin{array}{c}350 \mathrm{mg} \mathrm{Fe} \\
+ \text { Vitamin E } \\
\quad(7)\end{array}$ & $\begin{array}{c}1400 \mathrm{mg} \mathrm{Fe} \\
(8)\end{array}$ & $\begin{array}{c}1400 \mathrm{mg} \mathrm{Fe} \\
+ \text { Vitamin E } \\
(9)\end{array}$ \\
\hline $\mathrm{TC}(\mathrm{mg} / \mathrm{dl})$ & $89.26 \pm 4.95$ & $136.13 \pm 5.41$ & $91.22 \pm 5.60$ & $143.48 \pm 9.32$ & $93.31 \pm 11.61$ & $155.8 \pm 8.7$ & $96.33 \pm 6.23$ & $182.86 \pm 7.91$ & $103.25 \pm 9.26$ \\
\hline \multirow{2}{*}{$\%$ change } & & $52.51 \% \mathrm{c}$ & $2.2 \% \mathrm{c}$ & $60.74 \% \mathrm{c}$ & $4.54 \% \mathrm{c}$ & $74.54 \% \mathrm{c}$ & $7.92 \% \mathrm{c}$ & $104.86 \% \mathrm{c}$ & $15.67 \% \mathrm{c}$ \\
\hline & & & $-33 \% d$ & & $-34.96 \% \mathrm{~d}$ & & $-38.17 \% \mathrm{~d}$ & & $-43.53 \% \mathrm{~d}$ \\
\hline Significance & & $a^{* *}$ & N.S b** & $a^{* *}$ & N.S b** & $a^{* *}$ & $a^{*} b^{* *}$ & $a^{* *}$ & $a^{* *} b^{* *}$ \\
\hline TG (mg/dl) & $81.14 \pm 5.67$ & \begin{tabular}{|l|}
$95.61 \pm 6.67$ \\
\end{tabular} & $84.75 \pm 7.5$ & $107.03 \pm 9.41$ & $88.30 \pm 9.33$ & $124.10 \pm 7.79$ & $93.52 \pm 9.52$ & $142.57 \pm 7.8$ & $99.1 \pm 9.75$ \\
\hline \multirow{2}{*}{$\%$ change } & & $17.83 \% \mathrm{c}$ & $4.45 \% \mathrm{c}$ & $31.9 \% \mathrm{c}$ & $8.82 \% \mathrm{c}$ & $52.94 \% \mathrm{c}$ & $15.25 \% \mathrm{c}$ & $75.7 \% \mathrm{c}$ & $22.13 \% \mathrm{c}$ \\
\hline & & & $-11.36 \% \mathrm{~d}$ & & $-17.5 \% \mathrm{~d}$ & & $-24.64 \% \mathrm{~d}$ & & $-30.5 \% \mathrm{~d}$ \\
\hline Significance & & $a^{* *}$ & N.S b* & $a^{* *}$ & $a^{*} b^{* *}$ & $a^{* *}$ & $\mathrm{a}^{* *} \mathrm{~b}^{* *}$ & $a^{* *}$ & $a^{* *} b^{* *}$ \\
\hline HDL-C (mg/dl) & $52.2 \pm 6.92$ & $48.02 \pm 5.54$ & $51.67 \pm 6.15$ & $46.32 \pm 5.9$ & $48.32 \pm 5.49$ & $42.53 \pm 3.1$ & $46.95 \pm 2.26$ & $39.93 \pm 4.21$ & $45.58 \pm 8.62$ \\
\hline \multirow{2}{*}{$\%$ change } & & $-8 \% \mathrm{c}$ & $-1.01 \% \mathrm{c}$ & $-11.26 \% \mathrm{c}$ & $-7.43 \% \mathrm{c}$ & $-18.52 \% \mathrm{c}$ & $-10.06 \% \mathrm{c}$ & $-23.5 \% \mathrm{c}$ & $-12.68 \% \mathrm{c}$ \\
\hline & & & $7.6 \% \mathrm{~d}$ & & $4.32 \% \mathrm{~d}$ & & $10.4 \% \mathrm{~d}$ & & $14.1 \% \mathrm{~d}$ \\
\hline Significance & & $a^{*}$ & N.S b* & $a^{*}$ & N.S b* & $a^{* *}$ & $a^{*} b^{*}$ & $a^{* *}$ & $a^{*} b^{*}$ \\
\hline LDL-C (mg/dl) & $20.81 \pm 1.29$ & $49 \pm 3.66$ & $22.6 \pm 1.22$ & $55.66 \pm 1.08$ & $27.33 \pm 1.53$ & $68.72 \pm 1.4$ & $32 \pm 3.9$ & $94.5 \pm 1.78$ & $37.85 \pm 2.9$ \\
\hline \multirow{2}{*}{$\%$ change } & & $135.46 \% \mathrm{c}$ & $8.6 \% \mathrm{c}$ & $167.46 \% \mathrm{c}$ & $31.33 \% \mathrm{c}$ & $230.22 \% \mathrm{c}$ & $53.77 \% \mathrm{c}$ & $354.1 \% \mathrm{c}$ & $81.88 \% \mathrm{c}$ \\
\hline & & & $-53.87 \% \mathrm{~d}$ & & $-50.9 \% \mathrm{~d}$ & & $-53.43 \% d$ & & $-59.95 \% \mathrm{~d}$ \\
\hline Significance & & $a^{* *}$ & $a^{*} b^{* *}$ & $a^{* *}$ & $\mathrm{a}^{* *} \mathrm{~b}^{* *}$ & $a^{* *}$ & $a^{* *} b^{* *}$ & $a^{* *}$ & $a^{* *} b^{* *}$ \\
\hline VLDL-C (mg/dl) & $16.22 \pm 1.13$ & $19.12 \pm 1.33$ & $16.95 \pm 1.5$ & $21.4 \pm 1.88$ & $17.65 \pm 1.86$ & $24.81 \pm 1.56$ & $18.7 \pm 1.9$ & $28.51 \pm 1.56$ & $19.81 \pm 1.95$ \\
\hline \multirow{2}{*}{$\%$ change } & & $17.88 \% \mathrm{c}$ & $4.5 \% \mathrm{c}$ & $31.93 \% \mathrm{c}$ & $8.81 \% \mathrm{c}$ & $52.96 \% \mathrm{c}$ & $15.3 \% \mathrm{c}$ & $75.77 \% \mathrm{c}$ & $22.13 \% \mathrm{c}$ \\
\hline & & & $-11.35 \% \mathrm{~d}$ & & $-17.52 \% \mathrm{~d}$ & & $-24.63 \% \mathrm{~d}$ & & $-30.51 \% \mathrm{~d}$ \\
\hline Significance & & $a^{* *}$ & N.S b* & $a^{* *}$ & $a * b * *$ & $a^{* *}$ & $\mathrm{a}^{* *} \mathrm{~b}^{* *}$ & $a^{* *}$ & $\mathrm{a}^{* *} \mathrm{~b}^{* *}$ \\
\hline HDL-C/TC ratio & $0.58 \pm 0.06$ & $0.35 \pm 0.04$ & $0.56 \pm 0.07$ & $0.32 \pm 0.03$ & $0.54 \pm 0.06$ & $0.27 \pm 0.02$ & $0.48 \pm 0.08$ & $0.22 \pm 0.02$ & $0.44 \pm 0.06$ \\
\hline \multirow{2}{*}{$\%$ change } & & $-39.6 \% \mathrm{c}$ & $-3.45 \% \mathrm{c}$ & $-44.82 \% \mathrm{c}$ & $-6.9 \% \mathrm{c}$ & $-53.44 \% \mathrm{c}$ & $-17.24 \% \mathrm{c}$ & $-62.1 \% \mathrm{c}$ & $-24.14 \% \mathrm{c}$ \\
\hline & & & $60 \% \mathrm{~d}$ & & $68.75 \% \mathrm{~d}$ & & $77.8 \% \mathrm{~d}$ & & $100 \% \mathrm{~d}$ \\
\hline Significance & & $a^{* *}$ & N.S b** & $a^{* *}$ & N.S b** & $a^{* *}$ & $a^{*} b^{* *}$ & $a^{* *}$ & $a^{* *} b^{* *}$ \\
\hline $\begin{array}{l}\text { LDL-C/HDL-C } \\
\text { ratio }\end{array}$ & $0.4 \pm 0.06$ & $1.03 \pm 0.15$ & $0.44 \pm 0.05$ & $1.21 \pm 0.18$ & $0.54 \pm 0.06$ & $1.62 \pm 0.12$ & $0.68 \pm 0.08$ & $2.38 \pm 0.24$ & $0.8 \pm 0.2$ \\
\hline \multirow{2}{*}{$\% \overline{\text { change }}$} & & $157.5 \% \mathrm{c}$ & $10 \% \mathrm{c}$ & $202.5 \% \mathrm{c}$ & $35 \% \mathrm{c}$ & $305 \% \mathrm{c}$ & $70 \% \mathrm{c}$ & $495 \% \mathrm{c}$ & $100 \% \mathrm{c}$ \\
\hline & & & $-57.3 \% \mathrm{~d}$ & & $-55.3 \% \mathrm{~d}$ & & $-58 \% \mathrm{~d}$ & & $-66.4 \% \mathrm{~d}$ \\
\hline Significance & & $\mathrm{a}^{* *}$ & N.S b** & $a^{* *}$ & $a^{* *} b^{* *}$ & $a^{* *}$ & $\mathrm{a}^{* *} \mathrm{~b} * *$ & $a^{* *}$ & $a^{* *} b^{* *}$ \\
\hline
\end{tabular}

Data are represented as mean \pm S.D (8 rats / group).

a: indicates the statistical difference in comparison to the control group.

b: indicates the statistical difference between the four iron-supplemented groups $(2,4,6$ and 8$)$ and the four iron + vitamin E-supplemented groups $(3,5,7$ and 9$)$.

c: \% change compared to the control group.

d: \% change of the four iron-supplemented groups (2, 4, 6 and 8) compared to the four iron + vitamin E-supplemented groups (3, 5, 7 and 9)

$* *$ : $<<0.01$ : highly significant. *: $\mathrm{p}<0.05$ : significant. N.S: Non-significant. 
Lipid peroxidation level (plasma MDA concentration)

As indicated in Table 6, feeding iron-supplemented diets resulted in increased plasma MDA concentration, when compared to the control group. On the other hand, feeding iron+vitamin E-supplemented diets resulted in a remarkable decrease of plasma MDA concentration, in comparison to iron-overloaded groups.

\section{Antioxidant enzymes}

Table 6 shows that feeding iron-supplemented diets resulted in significant decrease in activities of erythrocyte SOD and plasma CAT, when compared to the control group. On the other hand, feeding iron+vitamin E-supplemented diets was found to cause a remarkable increase of erythrocyte SOD and plasma CAT activities, in comparison to iron-overloaded groups.

\section{Total antioxidant capacity}

As shown in Table 6, feeding iron-supplemented diets resulted in diminished plasma total antioxidant capacity, when compared to the control group. On the other hand, feeding iron+vitamin E-supplemented diets was found to cause a significant increase of plasma total antioxidant capacity, in comparison to iron-overloaded groups.

\section{Vitamin E}

As illustrated in Table 6, feeding iron-supplemented diets resulted in significant decrease in plasma vitamin $\mathrm{E}$ levels, when compared to the control group. On the other hand, feeding iron+vitamin E-supplemented diets was found to cause a significant increase of plasma vitamin $\mathrm{E}$ levels, in comparison to iron-overloaded groups.

\section{Histological studies}

As illustrated in Table 7 and Figure 1, feeding ironsupplemented diets resulted in liver tissue alterations, including fatty change of the hepatocytes, congestion of the hepatoportal blood vessel, Kupffer cells activation, fibrin thrombus and hepatic necrosis, when compared to the control group. On the other hand, feeding iron+vitamin E-supplemented diets was found to ameliorate iron overload-induced histological changes, in comparison to iron-overloaded groups.

Table 6: Levels of MDA, SOD, CAT, total antioxidant capacity and vitamin E of the study groups

\begin{tabular}{|c|c|c|c|c|c|c|c|c|c|}
\hline Parameters & $\begin{array}{c}\text { Control } \\
\text { (1) } \\
\end{array}$ & $\begin{array}{c}105 \mathrm{mg} \mathrm{Fe} \\
(2) \\
\end{array}$ & $\begin{array}{c}105 \mathrm{mg} \mathrm{Fe} \\
+ \text { Vitamin } \mathrm{E} \\
(3) \\
\end{array}$ & $\begin{array}{c}175 \mathrm{mg} \mathrm{Fe} \\
(4)\end{array}$ & $\begin{array}{c}175 \mathrm{mg} \mathrm{Fe} \\
+ \text { Vitamin E } \\
(5) \\
\end{array}$ & $\begin{array}{c}350 \mathrm{mg} \mathrm{Fe} \\
(6) \\
\end{array}$ & $\begin{array}{c}350 \mathrm{mg} \mathrm{Fe} \\
+ \text { Vitamin } \mathrm{E} \\
(7) \\
\end{array}$ & $\begin{array}{c}1400 \mathrm{mg} \mathrm{Fe} \\
(8) \\
\end{array}$ & $\begin{array}{c}1400 \mathrm{mg} \mathrm{Fe} \\
\text { +Vitamin E } \\
(9)\end{array}$ \\
\hline MDA (nmol/l) & $2.91 \pm 0.2$ & $3.21 \pm 0.44$ & $3.05 \pm 0.44$ & $3.58 \pm 0.45$ & $3.39 \pm 0.54$ & $4.38 \pm 0.35$ & $4.11 \pm 0.3$ & $8.13 \pm 0.28$ & $4.5 \pm 0.38$ \\
\hline \multirow{2}{*}{$\%$ change } & & $10.31 \% \mathrm{c}$ & $4.81 \% \mathrm{c}$ & $23.02 \% \mathrm{c}$ & $16.5 \% \mathrm{c}$ & $50.51 \% \mathrm{c}$ & $41.24 \% \mathrm{c}$ & $179.4 \% \mathrm{c}$ & $54.63 \% \mathrm{c}$ \\
\hline & & & $-5 \% \mathrm{~d}$ & & $-5.31 \% \mathrm{~d}$ & & $-6.16 \% \mathrm{~d}$ & & $-44.6 \% \mathrm{~d}$ \\
\hline Significance & & N.S & N.S & $\mathrm{a}^{* *}$ & $a^{*}$ & $\mathrm{a}^{* *}$ & $a^{* *}$ & $\mathrm{a}^{* *}$ & $\mathrm{a} * * \mathrm{~b} * *$ \\
\hline SOD (U) & $356.20 \pm 5.8$ & $349.6 \pm 1.98$ & $345.4 \pm 11.24$ & $337.90 \pm 4.95$ & $333.51 \pm 22.3$ & $244.07 \pm 8.88$ & $326.30 \pm 0.96$ & $\begin{array}{c}209.06 \pm 15.7 \\
3\end{array}$ & $320.4 \pm 2.7$ \\
\hline \multirow{2}{*}{$\%$ change } & & $-1.85 \% \mathrm{c}$ & $-3.03 \% \mathrm{c}$ & $-5.14 \% \mathrm{c}$ & $-6.37 \% \mathrm{c}$ & $-31.48 \% \mathrm{c}$ & $-8.4 \% \mathrm{c}$ & $-41.3 \% \mathrm{c}$ & $-10.05 \% \mathrm{c}$ \\
\hline & & & $-1.2 \% \mathrm{~d}$ & & $-1.3 \% \mathrm{~d}$ & & $33.7 \% \mathrm{~d}$ & & $53.25 \% \mathrm{~d}$ \\
\hline Significance & & N.S & N.S & $a^{*}$ & $a^{*}$ & $\mathrm{a}^{* *}$ & $a * b * *$ & $a^{* *}$ & $\mathrm{a} * \mathrm{~b} * *$ \\
\hline Catalase (U) & $423.1 \pm 4.51$ & $411.94 \pm 3.25$ & $418.74 \pm 4.12$ & $397.53 \pm 5.5$ & $414.26 \pm 3.46$ & $386.84 \pm 2.8$ & $410.55 \pm 4.15$ & $373.83 \pm 2.31$ & $405.67 \pm 1.46$ \\
\hline \multirow{2}{*}{$\%$ change } & & $-2.64 \% \mathrm{c}$ & $-1.03 \% \mathrm{c}$ & $-6.04 \% \mathrm{c}$ & $-2.1 \% \mathrm{c}$ & $-8.57 \% \mathrm{c}$ & $-2.96 \% \mathrm{c}$ & $-11.64 \% \mathrm{c}$ & $-4.12 \% \mathrm{c}$ \\
\hline & & & $1.65 \% \mathrm{~d}$ & & $4.21 \% \mathrm{~d}$ & & $13.88 \% \mathrm{~d}$ & & $8.51 \% \mathrm{~d}$ \\
\hline Significance & & N.S & N.S & $a^{*}$ & N.S & $a^{*}$ & N.S b* & $a^{*}$ & N.S b* \\
\hline $\begin{array}{l}\text { Total antioxidant } \\
\text { capacity }(\mathrm{mM} / \mathrm{l})\end{array}$ & $1.45 \pm 0.07$ & $1.35 \pm 0.07$ & $1.4 \pm 0.1$ & $1.35 \pm 0.13$ & $1.45 \pm 0.1$ & $1.26 \pm 0.08$ & $1.55 \pm 0.1$ & $1.21 \pm 0.05$ & $1.64 \pm 0.04$ \\
\hline \multirow{2}{*}{$\%$ change } & & $-7 \% \mathrm{c}$ & $-3.45 c$ & $-7 \% c$ & $0 \% \mathrm{c}$ & $-13.10 \% \mathrm{c}$ & $7 \% \mathrm{c}$ & $-16.55 \% \mathrm{c}$ & $13.1 \% \mathrm{c}$ \\
\hline & & & $3.7 \% \mathrm{~d}$ & & $7.4 \% \mathrm{~d}$ & & $23 \% \mathrm{~d}$ & & $35.5 \% \mathrm{~d}$ \\
\hline Significance & & $a^{*}$ & N.S & $\mathrm{a}^{*}$ & N.Sb* & $\mathrm{a}^{* * *}$ & $\mathrm{a}^{* *} \mathrm{~b}^{* *}$ & $\mathrm{a}^{* *}$ & $\mathrm{a} * * \mathrm{~b} * *$ \\
\hline Vitamin E $(\mu \mathrm{g} / \mathrm{dl})$ & $862 \pm 7.3$ & $774.13 \pm 9.8$ & $841.92 \pm 8$ & $732.7 \pm 8.6$ & $811.7 \pm 10$ & $681.8 \pm 7$ & $793 \pm 7.6$ & $602 \pm 11$ & $791 \pm 6.6$ \\
\hline \multirow{2}{*}{$\%$ change } & & $-10.2 \% \mathrm{c}$ & $-2.33 \% \mathrm{c}$ & $-15 \% \mathrm{c}$ & $-5.83 \% \mathrm{c}$ & $-20.9 \% \mathrm{c}$ & $-8 \% \mathrm{c}$ & $-30.16 \% \mathrm{c}$ & $-8.2 \% \mathrm{c}$ \\
\hline & & & $8.75 \% \mathrm{~d}$ & & $10.8 \% \mathrm{~d}$ & & $16.3 \% \mathrm{~d}$ & & $31.4 \% \mathrm{~d}$ \\
\hline Significance & & $\mathrm{a}^{* *}$ & N.S b** & $\mathrm{a}^{* *}$ & $a * b * *$ & $\mathrm{a}^{* *}$ & $a * b * *$ & $a^{* *}$ & $a * b * *$ \\
\hline
\end{tabular}

Data are represented as mean \pm S.D ( 8 rats / group).

a: indicates the statistical difference in comparison to the control group.

b: indicates the statistical difference between the four iron-supplemented groups $(2,4,6$ and 8$)$ and the four iron + vitamin E-supplemented groups $(3,5,7$ and 9$)$.

c: \% change compared to the control group.

$\mathrm{d}$ : \% change of the four iron-supplemented groups (2, 4, 6 and 8) compared to the four iron + vitamin E-supplemented groups (3, 5, 7 and 9)

**: $\mathrm{p}<0.01$ : highly significant. *: $\mathrm{p}<0.05$ : significant. N.S: Non-significant. 
Table 7: Liver histological analysis of the study groups

\begin{tabular}{|c|c|c|c|c|c|c|c|c|c|}
\hline$\overbrace{\text { Parameters }}^{\text {Groups }}$ & $\begin{array}{c}\text { Control } \\
\text { (1) }\end{array}$ & $\begin{array}{c}105 \mathrm{mg} \mathrm{Fe} \\
\text { (2) }\end{array}$ & $\begin{array}{c}105 \mathrm{mg} \mathrm{Fe} \\
+ \text { Vitamin } \mathrm{E} \\
\text { (3) }\end{array}$ & $\begin{array}{c}175 \mathrm{mg} \mathrm{Fe} \\
\text { (4) }\end{array}$ & $\begin{array}{c}175 \mathrm{mg} \mathrm{Fe} \\
+ \text { Vitamin } \mathrm{E} \\
\quad(5)\end{array}$ & $\begin{array}{c}350 \mathrm{mg} \mathrm{Fe} \\
\text { (6) }\end{array}$ & $\begin{array}{c}350 \mathrm{mg} \mathrm{Fe} \\
+ \text { Vitamin } \mathrm{E} \\
\quad(7)\end{array}$ & $\begin{array}{c}1400 \mathrm{mg} \mathrm{Fe} \\
(8)\end{array}$ & $\begin{array}{l}1400 \mathrm{mg} \mathrm{Fe} \\
+ \text { Vitamin } \mathrm{E} \\
\text { (9) }\end{array}$ \\
\hline $\begin{array}{l}\text { Normal histological } \\
\text { structure of the } \\
\text { hepatic lobule }\end{array}$ & (+ve) $4 / 4$ & $(+v e) 4 / 4$ & $(+v e) 4 / 4$ & (-ve) $4 / 4$ & (-ve) $4 / 4$ & $(-\mathrm{ve})$ 4/4 & (-ve) $4 / 4$ & (-ve) $4 / 4$ & (-ve) $4 / 4$ \\
\hline $\begin{array}{l}\text { Fatty change of the } \\
\text { hepatocytes }\end{array}$ & $(-\mathrm{ve}) 4 / 4$ & $(-v e) 4 / 4$ & $(-\mathrm{ve}) 4 / 4$ & $\begin{array}{l}\text { (+ve) } 2 / 4 \\
(-v e) 2 / 4\end{array}$ & $(-v e) 4 / 4$ & $(+v e) 4 / 4$ & (-ve) $4 / 4$ & $(+v e) 4 / 4$ & $\begin{array}{l}(+v e) 1 / 4 \\
(-v e) 3 / 4\end{array}$ \\
\hline $\begin{array}{l}\text { Congestion of the } \\
\text { hepatoportal } \\
\text { blood vessel }\end{array}$ & (-ve) $4 / 4$ & $(-\mathrm{ve})$ 4/4 & $(-v e) 4 / 4$ & $\begin{array}{l}(+v e) 3 / 4 \\
(-v e) 1 / 4\end{array}$ & $(-\mathrm{ve}) 4 / 4$ & $(+v e) 4 / 4$ & (-ve) $4 / 4$ & $(+v e) 4 / 4$ & $\begin{array}{l}(+v e) 1 / 4 \\
(-v e) 3 / 4\end{array}$ \\
\hline $\begin{array}{l}\text { Kupffer cells } \\
\text { activation }\end{array}$ & $(-\mathrm{ve}) 4 / 4$ & $(-\mathrm{ve}) 4 / 4$ & (-ve) $4 / 4$ & $\begin{array}{l}(+v e) 3 / 4 \\
(-v e) 1 / 4 \\
\end{array}$ & (-ve) $4 / 4$ & $\begin{array}{l}(+v e) 3 / 4 \\
(-v e) 1 / 4 \\
\end{array}$ & (-ve) $4 / 4$ & $(+v e) 4 / 4$ & (-ve) $4 / 4$ \\
\hline Fibrin thrombus & $(-\mathrm{ve}) 4 / 4$ & $(-v e) 4 / 4$ & (-ve) $4 / 4$ & (-ve) $4 / 4$ & (-ve) $4 / 4$ & $(-v e) 4 / 4$ & (-ve) $4 / 4$ & $\begin{array}{l}\text { (+ve) } 2 / 4 \\
(-v e) 2 / 4\end{array}$ & (-ve) $4 / 4$ \\
\hline Hepatic necrosis & $(-\mathrm{ve}) 4 / 4$ & $(-v e) 4 / 4$ & $(-v e) 4 / 4$ & $(-v e)$ 4/4 & $(-v e)$ 4/4 & (-ve) $4 / 4$ & $(-\mathrm{ve}) 4 / 4$ & $(+v e) 4 / 4$ & $(-v e)$ 4/4 \\
\hline
\end{tabular}

Histological changes are represented as the number of the affected sections divided by the number of the examined sections.
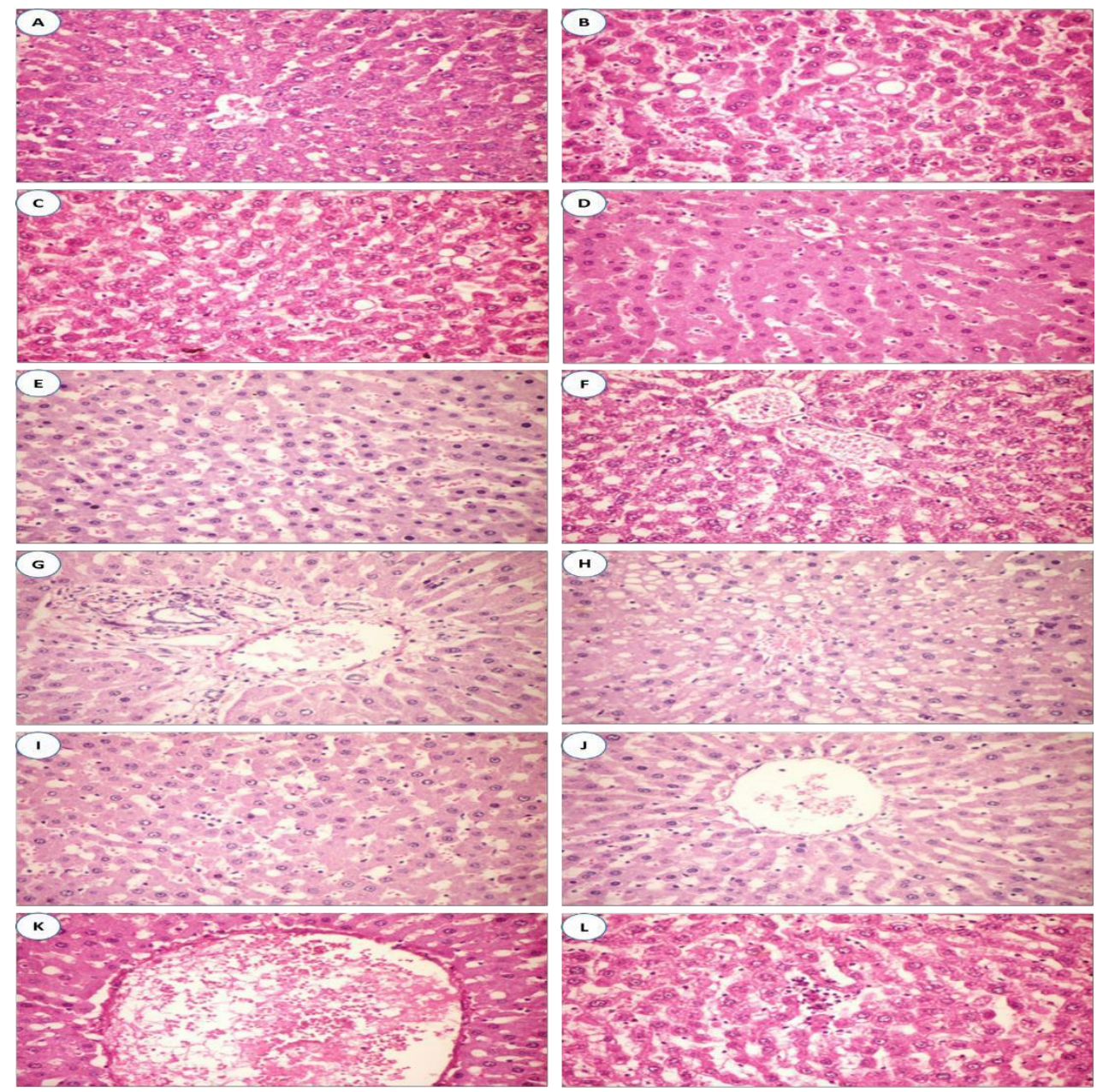

Fig 1: Histological examination of liver sections from the study groups stained with hematoxylin and eosin. (A) Control group showing the normal histological structure of the hepatic lobule. (B) Group 4 (fed on $175 \mathrm{mg}$ iron) showing fatty change of the hepatocytes. (C) Group 6 (fed on $350 \mathrm{mg}$ iron) showing fatty change of the hepatocytes. (D) Group 4 (fed on $175 \mathrm{mg}$ iron) showing congestion of the hepatic sinusoids. (E) Group 6 (fed on $350 \mathrm{mg}$ iron) showing congestion of the hepatic sinusoids. (F) Group 4 (fed on $175 \mathrm{mg}$ iron) showing kupffer cells activation. (G) Group 6 (fed on $350 \mathrm{mg}$ iron) showing kupffer cells activation. (H) Group 8 (fed on $1400 \mathrm{mg}$ iron) showing fatty change of the hepatocytes. (I) Group 8 (fed on $1400 \mathrm{mg}$ iron) showing congestion of the hepatic sinusoids. (J) Group 8 (fed on $1400 \mathrm{mg}$ iron) showing kupffer cells activation. (K) Group 8 (fed on 1400 $\mathrm{mg}$ iron) showing fibrin thrombus. (L) Group 8 (fed on $1400 \mathrm{mg}$ iron) showing hepatic necrosis. 


\section{Discussion}

The significant decrease in body weight gain in the iron overloaded groups in the present study is in agreement with a previous study ${ }^{[29]}$, which reported that iron overload caused a significant decrease in body weight gain in comparison to the control group. In support of the present results, a previous study [9], found that feeding experimental animals carbonyl iron supplemented diets with $35,350,3500$ and $20.000 \mu \mathrm{g}$ iron/g diet for 12 weeks caused a significant decrease in body weight in all studied groups as compared to the control group. In the same line, Lou et al., induced iron overload by intraperitoneal injection of iron dextran and reported that body weight was significantly lower in iron overloaded rats than in normal controls ${ }^{[30}$.

Furthermore, Reardon and Allen, declared that induction of iron overload in mice leads to $21 \%$ decrease in body weight gain in comparison to the control group ${ }^{[31]}$.

On the other hand, our results disagree with those of Fischer et al., who reported that on feeding rats iron overload diets containing $20,45,650,1500 \mathrm{mg} / \mathrm{kg}$ diet for 28 days, body weight gain did not altered by dietary iron concentration ${ }^{[32]}$. Meanwhile, a previous study declared that induction of iron overload using $10 \mathrm{mg}$ iron dextran/day for 5 days did not affect final body weight or weight gain. However, absolute and relative liver weights were increased by iron dextran injection ${ }^{[33]}$. Moreover, it has been reported that food intake in iron overloaded rats did not change by feeding $1500 \mathrm{mg}$ iron/kg diet in comparison to the control rats ${ }^{[34]}$.

The relative improvement in body weight gain, food intake and feed efficiency ratio in the present study is in agreement with the findings of a previous study, which reported that vitamin $\mathrm{E}$ was found to improve daily food intake, body weight gain and feed efficiency ratio in copper and lead intoxicated male albino rats ${ }^{[35]}$. Additionally, it has been demonstrated that vitamin $\mathrm{E}$ had a relatively more profound effect on the skeletal muscles than other tissues and that skeletal muscles is the most sensitive tissue to dietary vitamin $\mathrm{E}$ status, with necrotizing myopathy being the most common sign of vitamin $\mathrm{E}$ deficiency in many species of animals. Interestingly, the relatively great suppressing effect of vitamin $\mathrm{E}$ on the levels of labile iron and lipid peroxidation products in the muscles explains the partial correction of body weight upon treatment with vitamin $\mathrm{E}^{[36]}$.

It has been declared that induction of iron overload in mice resulted in a lower growth rate. Additionally, the weight of liver, heart and spleen of iron-overloaded mice was found to be increased in comparison to the control group. Furthermore, it has been demonstrated that iron plays an integral part in the progression of hepatic fibrosis via its ability to catalyze the formation of highly reactive and damaging ROS which initiate lipid peroxidation, protein oxidation and DNA modification, leading ultimately to apoptosis and necrosis ${ }^{[37]}$.

Many investigators ${ }^{[38,39]}$, have described the antioxidant effect of vitamin E, which could ameliorate the effect of
ROS on the liver, thereby restoring its normal weight in comparison to the control group.

The decrease in hemoglobin and hematocrit levels in the iron overloaded rats in the current study is in line with the results of the study of Flora ${ }^{[40]}$, who declared that the red blood cell indices can be an indicator of oxidative status, because erythrocytes are one of the major production sites of free radicals and some of them can trigger peroxidation of fatty acids in their membrane phospholipids, thereby altering their quality (integrity, size and quantity).

The therapeutic use of antioxidant vitamins has gained considerable interest during the last decade. Previous animal and human studies showed that vitamin E might play an important role in preventing the oxidation of polyunsaturated fatty acids in erythrocytes membrane, thereby maintaining erythrocytes membrane integrity and inhibiting oxidative stress which leads to erythrocytes lysis ${ }^{[41]}$. Additionally, vitamin E was known to be the most effective natural lipid soluble chain-breaking antioxidant which scavenges free radicals, thereby preventing free radicals generation and reduce lipid peroxidation in erythrocytes membrane, resulting in decreased erythrocytes hemolysis and normalization of all hematological parameters ${ }^{[42]}$.

It has been previously demonstrated that treatment with vitamin $\mathrm{E}$ was found to increase the number of colony forming units of erythroid precursors and enhance erythropoiesis, hemoglobin levels, thereby corrected the experimentally-induced anemia ${ }^{[43]}$.

Vitamin E was found to treat and/or prevent anemia by inhibiting polyunsaturated fatty acids oxidation in erythrocytes membrane, thereby reducing the fragility of erythrocytes and preventing the oxidative stress-induced erythrocytes lysis ${ }^{[44]}$. Alternatively, vitamin $\mathrm{E}$ was found to enhance erythropoiesis, therapy improving blood hemoglobin and hematocrit levels ${ }^{[45]}$.

In the present study, in the iron overloaded rats, in all groups there were a significant increase in plasma iron, ferritin and transferrin saturation, with a significant decrease in the total iron binding capacity, in comparison to the control group. Our results are in agreement with those of Junge et al., ${ }^{[46]}$ who reported that iron overload induced a significant increase in serum iron levels in experimental animals.

Also, it has been declared that, in iron overload, the storage and transport proteins such as ferritin and transferrin become saturated. Thus, iron spills over into other tissues and organs ${ }^{[47]}$. In the same line, Zhang et al., ${ }^{[48]}$ reported that serum iron was elevated significantly in iron overloaded mice.

Furthermore, Nahdi et al., ${ }^{[49]}$ found that serum iron was significantly increased in iron overloaded rats in comparison to the control rats. They also reported that transferrin saturation was more than $100 \%$ with the presence of non-transferrin bound iron in iron overloaded rats when compared to the control rats.

Regarding plasma ferritin concentration, it has been reported that serum ferritin concentration is the most 
commonly used indirect estimate of body iron store ${ }^{[50]}$. Moreover, excess iron was found to regulate the synthesis of the iron storage protein ferritin by translational mechanism ${ }^{[51]}$.

Results of the present study are in agreement with those obtained by Torti and Torti, ${ }^{[52]}$ who reported that iron treatment was found to be associated with increased basal levels of ferritin ${ }^{[55]}$. Also, It has been observed that ferritin levels were significantly elevated in iron overloaded mice as compared to the control rats ${ }^{[47]}$. Additionally, Zhang et al., confirmed that iron-dextran overloading significantly increased the serum ferritin level in mice ${ }^{[48]}$.

In the current study, the significant decrease in iron profile in iron overloaded rats upon vitamin $\mathrm{E}$ supplementation could be explained by enhancing erythropoiesis. It has been previously reported that treatment with vitamin $\mathrm{E}$ resulted in increased number of colony forming units of erythroid precursors, enhanced erythropoiesis and improved hemoglobin levels ${ }^{[45]}$. In addition, vitamin $E$ might be resulted in the reduction of labile iron. It has been reported that vitamin $\mathrm{E}$ was found to reduce the rate of mitochondrial superoxide generation as well as levels of labile iron and lipid peroxidation products in a dose-dependent manner. In addition, it has been suggested that vitamin $\mathrm{E}$ may exert its antioxidant function by limiting the generation and/or levels of superoxide radicals ${ }^{[36]}$. Furthermore, it has been suggested that vitamin E not only reduces the levels of harmful free radicals but also attenuates the release of iron from iron- transport and/or storage proteins ${ }^{[53]}$. It has been previously concluded that dietary vitamin $\mathrm{E}$ may protect against oxidative tissue damage by reducing the generation of superoxide radicals, thereby attenuating the release of iron from its protein complexes ${ }^{[54]}$.

Furthermore, vitamin $\mathrm{E}$ is known to prevent tissue destruction and iron release. It has been reported that oxidative stress caused by insufficient vitamin $\mathrm{E}$ intake might leads to the damage of iron-containing cellular components, thereby increasing iron release ${ }^{[36]}$.

In the present study, iron overloaded-rats in all groups exhibited significant increases in plasma ALT and AST activities when compared to the control rats. In the current study results are in line with that reported by Asare et al., who showed that iron supplemented-rats revealed a significant increase in ALT activity as compared to the control rats ${ }^{[56]}$. Additionally, Silva et al., ${ }^{[55]}$ observed that the administration of iron dextran to rats resulted in increased AST activity. Furthermore, it has been declared that the increase in activities of serum liver enzymes in iron overloaded-rats might be attributed to the generation of ROS and subsequent oxidative damage caused by excess hepatic iron ${ }^{[40]}$.

In the current study, vitamin $\mathrm{E}$ supplemented-rats showed significant decrease in plasma ALT and AST activities as compared to the iron- overloaded rats. Data are in line with Hashemian et al., ${ }^{[57]}$ who reported that vitamin $\mathrm{E}$ was found to improve the liver function have as well as the antioxidant status.

In the present study, in iron overloaded groups, a significant increase in the plasma TC, TG, LDL-C and VLDL-C levels was observed when compared to the control group. It has been suggested that iron-induced excessive lipid peroxidation resulted in alterations of lipid metabolism, possibly by direct/indirect effect on lipogenesis-related genes and key enzymes of cholesterol homeostasis. In addition, oxidative changes of lipoproteins may leads to altered lipoprotein-receptor interactions in the extra-hepatic target cells, resulting in alterations of the lipid profile. Furthermore, the oxidative damage of the liver was found to induce alterations in the cholesterol biosynthetic pathways, resulting in increased serum TC levels with concurrent increases in serum phospholipids as reported by Wittaker et al ${ }^{[9]}$.

Vitamin E is emerged as an essential fat soluble nutrient that functions as an antioxidant. It is essential because the body can not manufacture its own vitamin E, foods and supplements must provide it. Vitamin E represents one of the most fascinating natural resources that have the potential to influence a broad range of mechanisms

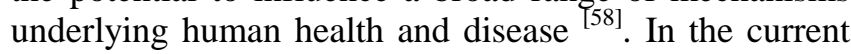
study supplementation of vit.E to the iron loaded diets resulted in enhancement in plasma lipid variables exhibited from the significant reductions in plasma TC, TG, LDL-C and VLDL-C together with a significant elevations in plasma HDL-C levels compared to iron loaded diets without vit.E supplementation.

Our results are in line with those reported by Achuba, who studied the protective role of vitamins $\mathrm{E}$ and $\mathrm{C}$ on petroleum contaminated diet-induced oxidative stress in rabbits. In regards to lipid profile, TC and LDL-C levels were found to be increased, while HDL-C levels were found to be decreased in comparison to rabbits fed on standard diet. Oxidative stress induction was indicated by significant increase of lipid peroxidation levels which was accompanied by a non-significant decrease in superoxide dismutase and catalase activities. Feeding rabbits on diets containing vitamins $\mathrm{E}$ and $\mathrm{C}$ resulted in normalization of the lipid profile variables as well as amelioration of lipid peroxidation ${ }^{[59]}$.

The increase of MDA levels in iron overloaded-rats is in line with a previous study, which reported that the prooxidant action of the catalytically active iron is primarily related to its ability to promote lipid peroxidation, and to catalyze the generation of highly cytotoxic hydroxyl radicals from hydrogen peroxide via Fenton or HaberWeiss reactions [60]. Furthermore, it has been demonstrated that iron-mediated increase of MDA levels was found to be associated with iron-induced lipid peroxidation with subsequent oxidative damage which are considered as well-known features of iron toxicity ${ }^{[61]}$. The presented data revealed a decrease of MDA levels in vitamin E-supplemented groups when compared to the iron- overloaded groups. In line with our results, it has been reported that vitamin $\mathrm{E}$ was found to transfer its phenolic hydrogen to a peroxyl free radical of a peroxidized polyunsaturated fatty acid, thereby breaking 
the radical chain reaction, preventing further polyunsaturated fatty acids peroxidation in cellular and subcellular membrane phospholipids, and subsequently resulted in a decreased MDA levels ${ }^{\text {[62] }}$

Under normal cellular metabolism, endogenous antioxidant protection consists of cytoplasmic enzymes such as SOD and CAT. It is known that SOD catalyzes the dismutation of superoxide radicals to hydrogen peroxide which is transformed into water by CAT ${ }^{\text {63] }}$. Accordingly, ROS generated during normal cellular processes are immediately detoxified by the endogenous antioxidant enzymes such as CAT and SOD ${ }^{[64]}$. In the present study, there was a significant decrease in erythrocytes SOD and plasma CAT activities in iron overloaded-rats when compared to the control rats. The presented findings are in accordance with those of Zhao et al. ${ }^{[47]}$ who showed that iron-dextran injection in rats caused a significant decrease in hepatic CAT and SOD activities ${ }^{[49]}$. In the current study, there was a significant increase in erythrocytes SOD and plasma CAT activities in vitamin E-supplemented groups when compared to the iron-overloaded groups. In line with our data, Koyu et al. ${ }^{[65]}$ reported that induction of iron overload using iron dextran causes a significant decrease in erythrocytes SOD and CAT activities. On the other hand, upon vitamin E supplementation, erythrocytes SOD and CAT activities were found to be significantly increased.

In the same line, it has been suggested that supplementation with vitamin $\mathrm{E}$ was found to improve plasma total antioxidant status, confirming the antioxidant properties of vitamin $\mathrm{E}^{[66]}$.

In the present study, the decrease of the plasma total antioxidant capacity in iron overloaded-groups might be attributed to iron overload-mediated imbalance between pro-oxidants and antioxidants which resulted in a severe loss of endogenous antioxidants.

It is known that antioxidant enzymes such as SOD and CAT operate in conjunction with several non-enzymatic molecules to contrast the ROS actions and to avoid oxidative damage ${ }^{[67]}$. These enzymes significantly contribute to the total antioxidant capacity and are the first line of defense against oxidative stress ${ }^{[68]}$.

In the current study, the increase of the plasma total antioxidant capacity noticed in vitamin E-supplemented groups when compared to the iron-overloaded groups could be necessary to detoxify increased lipid peroxidation as described by Hunderkari ${ }^{[69]}$.

Taking into account earlier research acknowledged antioxidants inhibition in response to oxidative stress, it has been suggested that a preliminary depletion of total antioxidant capacity is induced by oxidative stress but is compensated by the release and activation of stock enzymatic antioxidants from their stores such as liver and adipose tissue ${ }^{[70]}$.

In the presented study, the reduction in plasma vitamin E concentrations in iron overloaded-rats as compared to the control group may be attributed to oxidative stress which might caused a significant depletion of endogenous vitamins E stores.
In the current study, there was a significant increase in plasma vitamin $\mathrm{E}$ level in vitamin E-supplemented groups when compared to the iron- overloaded groups. These findings could be explained by the ability of vitamin $\mathrm{E}$ supplementation to restore normal vitamin $\mathrm{E}$ levels.

In the present study, there were pathological signs in the histological examination of liver tissues from iron overloaded-rats represented by fatty changes of hepatocytes, congestion of hepatoportal blood vessel, kupffer cell activation, fibrin thrombus in central vein and hepatic necrosis.

It has been suggested that serum iron and ferritin levels might be correlated with liver inflammation and serum markers of fibrogenesis. Accordingly, increased iron stores may stimulate hepatic fibrogenesis by oxygen free radicals and induce the production of fibrogenic cytokines ${ }^{[71]}$. These data may explain the improvement of hepatic fibrosis upon antioxidant therapy, which decreases the oxidative stress markers with subsequent decrease in the hepatic fibrogenesis stimuli.

It has been found that chronic iron overload in a murine model leads to a significant dose dependent increase in total hepatic iron burden with a corresponding increase in MDA levels and a significant decrease in plasma vitamin E levels. These findings suggested that ironcatalyzed oxygen free radicals generation might be implicated in liver tissue damage upon chronic iron overload $^{[37]}$.

It has been demonstrated that lipid peroxidation in hepatocytes is the key pathogenic event leading hepatic necrosis. At this point two factors may occur, possibly together. First, the release of lipid peroxidation products from necrotic hepatocytes and/or phagocytosis of necrotic hepatocytes debris may stimulate activation of kuppfer cells, which in turn release soluble factors that activate stellate cells. Second, the necrotic hepatocytes may themselves release growth factors and cytokines that are mitogenic (and possibly fibrogenic) for stellate cells ${ }^{[72]}$.

It has also been described that Kupffer cells are the first cells exposed to materials absorbed from the gastrointestinal tract. Their ability to eliminate and detoxify microorganisms, endotoxins, degenerated cells, immune complexes and toxic agents is an important physiological function. Furthermore, the pivotal role of kupffer cells in the initiation of hepatocellular damage is supported by experimental models that have demonstrated a correlation between the degree of activation of Kupffer cells and the degree of hepatocellular destruction ${ }^{[72]}$.

Noteworthy, free radical formation and the generation of lipid peroxidation products, due to excess iron resulted in the induction of oxidative stress through an increased rate of hydroxyl radical generation by the Haber-Weiss reaction, and may result in progressive tissue injury as fibrosis eventually cirrhosis or hepatocellular carcinoma [73]. 
The histological examination of liver tissue from vitamin E-supplemented rats revealed an obvious ameliorating effect against iron overload side effects. As free radicals generation and lipid peroxidation are the proposed mechanisms of iron-induced liver toxicity, vitamin E supplementation was found to exert a hepatoprotective role through free radicals scavenging actions which were concomitant with a significant decrease in MDA production. The improvement in histopathological findings following treatment of vitamin $\mathrm{E}$ is in agreement with the observations of a previous study ${ }^{[38]}$, which reported that vitamin $\mathrm{E}$ may protect against oxidative tissue damage by reducing the generation and/or level of superoxide, which in turn attenuate the release of iron from its protein complexes.

Among the four iron overloaded levels the $1400 \mathrm{mg}$ level responds mostly by the vitamin $\mathrm{E}$ dose (375 $\mathrm{IU} / \mathrm{Kg}$ ).

\section{Conclusions}

In conclusion, vitamin $\mathrm{E}$ supplementation may be useful for ameliorating iron overload-induced side effects via ROS scavenging-mediated mechanisms, thereby modulating lipids peroxidation, increasing antioxidant enzyme activities and improving total antioxidant capacity.

\section{References}

1) Steinbicker, A. U. and Muchkenthaler, M. U. (2013). Out of balance-systemic iron homeostasis in iron-related disorders. Nutrients. 5(8): 3034-3061.

2) Ferrari, M., Mistura, L., Patterson, E., Sjöström, M., Díaz, L. E., Stehle, P., Gonzalez-Gross, M., Kersting, M., Widhalm, K., Molnár, D., Gottrand, F., De, Henauw, S., Manios, Y., Kafatos, A., Moreno, L. A., Leclercq, C. and HELENA study group (2011). Evaluation of iron status in European adolescents through biochemical iron indicators: the HELENA Study. Eur. J. Clin. Nutr. 65(3): 340-349.

3) Day, S. M., Duquaine, D., Mundada, L. V., Menon R. G., Khan, B. V., Rajagopalan, S. and Fay, W. P. (2003). Chronic iron administration increases vascular oxidative stress and accelerates arterial thrombosis. Circulation, 107(20):2601-2606.

4) Zhang, D. L., Ghosh, M. C. and Rouault, T. A. (2014). The physiological functions of iron regulatory proteins in iron homeostasis - an update. Front Pharmacol. 5: 124.

5) Allen, L., de Benoist, B., Dary, O. and Hurrel, R. (2006). Guidelines on food fortification with micronutrients. World Health Organization and Food and Agriculture Organization of the United Nations. Available from: http://www.who.int/nutrition/publications/guide_foo d_fortification_micronutrients.pdf.

6) Pruthi, S., Allison, T. and Hensrud, D. (2001). Vitamin E supplementation in the prevention of coronary heart diseases. Mayo Clin. Proc. 76(11): $1131-1136$.
7) Carrier, J., Aghdassi, E., Cullen, J. and Allard, J. P. (2002). Iron supplementation increases disease activity and vitamin $\mathrm{E}$ ameliorates the effect in rats with dextran sulfate sodium-induced colitis. J. Nutr. 132(10): 3146-3150.

8) Reeves, P. G., Nielsen, F. H. and Fahey, G. C. Jr. (1993). AIN-93 purified diets for laboratory rodents: final report of the American Institute of Nutrition ad hoc writing committee on the reformulation of the AIN-76A rodent diet. J. Nutr. 123(11): 1939-1951.

9) Whittaker, P. and Chanderbhan, R. F. (2001). Effect of increasing iron supplementation on blood lipids in rats. Br. J. Nutr. 86(5): 587-592.

10) Chapman, D. G., Castillo, R. and Campbell, J. A. (1959). Evaluation of protein in food. I. A method for the determination of protein efficiency ratios. Can. J. Biochem. Physiol. 37(5): 679-686.

11) Drabkin, D. L. and Austin, J. H. (1935). Spectrophotometric studies: II. Preparations from washed blood cells; nitric oxide hemoglobin and sulfhemoglobin. J. Biol. Chem. 112: 51-56.

12) McInroy, R. A. (1954). A micro-haematocrit for determining the packed cell volume and haemoglobin concentration on capillary blood. J. Clin. Path. 7(1): 32-36.

13) Stookey, L. L. (1970). Ferrozine, a new spectrophotometric reagent for iron. Anal. Chem. 42(7): 779-781.

14) Persijn, J. P., van der Slik, W. and Riethorst, A. (1971). Determination of serum iron and latent ironbinding capacity (LIBC). Clin. Chim. Acta. 35(1): 91-98.

15) Tomatsu, S, Orii, K. O., Fleming, R. E., Holden, C. C., Waheed, A., Britton, R. S., Gutierrez, M. A., Velez-Castrillon, S., Bacon, B. R. and Sly, W. S. (2003). Contribution of the H63D mutation in HFE to murine hereditary hemochromatosis. Proc. Natl. Acad. Sci. U. S. A. 100(26): 15788-15793.

16) Hunter, J. E. (1978). Variable effects of iron status on the concentration of ferritin in rat plasma, liver, and spleen. J. Nutr. 108(3): 497-505.

17) Reitman, S. and Frankel, S. (1957). A colorimetric method for the determination of serum glutamic oxalacetic and glutamic pyruvic transaminases. Am. J. Clin. Pathol. 28(1): 56-63.

18) Kind, P. R. N. and King, E. J. (1954). Estimation of Plasma Phosphatase by Determination of Hydrolysed Phenol with Amino-antipyrine. J. Clin. Pathol. 7(4): 322-326.

19) Richmond, W. (1973). Preparation and properties of a cholesterol oxidase from Nocardia sp. and its application to the enzymatic assay of total cholesterol in serum. Clin. Chem. 19(12): 1350-1356.

20) Koditschek, L. K. and Umbreit, W. W. (1969). Alpha-glycerophosphate oxidase in Streptococcus faecium F 24. J Bacteriol. 98(3): 1063-1068.

21) Burstein M, Scholnick HR and Morfin R. (1970). Rapid method for the isolation of lipoproteins from 
human serum by precipitation with polyanions. J. Lipid Res. 11(6): 583-595.

22) Friedewald, W. T., Levy, R. I. and Fredrickson, D. S. (1972). Estimation of the concentration of lowdensity lipoprotein cholesterol in plasma, without use of the preparative ultracentrifuge. Clin. Chem. 18: 499-502.

23) Uchiyama, M. and Mihara, M. (1978). Determination of malonaldehyde precursor in tissues by thiobarbituric acid test. Anal. Biochem. 86(1): 271-278.

24) Winterbourn, C. C., Hawkins, R. E., Brian, M. and Carrell, R. W. (1975). The estimation of red cell superoxide dismuatse activity. J. Lab. Clin. Med. 85(2): 337-341.

25) Maehly, A. C. and Chance, B. (1954). The assay of catalases and peroxidases. Methods Biochem. Anal. 1: $357-424$.

26) Koracevic, D., Koracevic, G., Djordjevic, V., Andrejevic, S. and Cosic, V. (2001). Method for the measurement of antioxidant activity in human fluids. J. Clin. Pathol. 54(2): 356-361.

27) Bieri, J. G., Tolliver, T. J. and Catignani, G. L. (1979). Simultaneous determination of alphatocopherol and retinol in plasma or red cells by high pressure liquid chromatography. Am. J. Clin. Nutr. 32(10): 2143-2149.

28) Bancroft, J. D., Stevens, A. and Turner, D. R. (1996). Theory and practice of histological techniques. 4th edition. New York: Churchill Livingstone.

29) Chen, K., Suh, J., Carr, A. C., Morrow, J. D., Zeind, J. and Frei, B. (2000). Vitamin C suppress oxidative lipid damage in vivo, even in the presence of iron overload. Am. J. physiol. Endocrinol. Metab. 279(6): E1406-1412.

30) Lou, L. X., Geng, B., Chen, Y., Yu, F., Zhao, J. and Tang, C. S. (2009). Endoplasmic reticulum stress involved in heart and liver injury in ironloaded rats. Clin. Exp. Pharmacol. Physiol. 36(7): 612-618.

31) Reardon, T. F. and Allen, D. G. (2009). Iron injections in mice increase skeletal muscle iron content, induce oxidative stress and reduce exercise performance. Exp. Physiol. 94(6): 720-730.

32) Fischer, J. G., Glauert, H. P., Yin, T., SweeneyReeves, M. L., Larmonier, N. and Black, M. C. (2002). Moderate iron overload enhances lipid peroxidation in livers of rats, but does not affect NFkappaB activation induced by the peroxisome proliferator, Wy-14,643. J. Nutr. 132(9): 25252531.

33) Turbino-Ribeiro, S. M., Silva, M. E., Chianca, D. A. Jr, De Paula, H., Cardoso, L. M., Colombari, E. and Pedrosa, M. L. (2003). Iron overload in hypercholesterolemic rats affects iron homeostasis and serum lipids but not blood pressure. J. Nutr. 133(1): 15-20.
34) Cockell, K. A., Wotherspoon, A. T., Belonje, B., Fritz, M. E., Madère, R., Hidiroglou, N., Plouffe, L. J., Ratnayake, W. M. and Kubow, S. (2005). Limited effect of combined dietary copper deficiency/iron overload and oxidative stress parameters in rat liver and plasma. J. Nutr. Biochem. 16(12): 750-756.

35) Osfor, M. M. H., Ibrahim, H., Mohamed, Y. A., Ahmed, S. M., Abd El Azeem, A. S. and Hegazy, A. M. (2010). Effect of alpha lipoic acid and vitamin $\mathrm{E}$ on heavy metals intoxication in male albino rats. $\mathrm{J}$. Am. Sci. 6(8): 56-63.

36) Ibrahim, W. and Chow, C. K. (2005). Dietary vitamin $\mathrm{E}$ reduces labile iron in rat tissues. Journal of Biochemical and Molecular Toxicology. 19(5): 298303.

37) McCullough, K. D. and Bartfay, W. J. (2007). The dose-dependent effects of chronic iron overload on the production of oxygen free radicals and vitamin $\mathrm{E}$ concentrations in the liver of a murine model. Biol. Res. Nurs. 8(4): 300-304.

38) Singal, A. K., Jampana, S. C. and Weinman, S. A. (2011). Antioxidants as therapeutic agents for liver disease. Liver Int. 31(10): 1432-1448.

39) Jiang, Q. (2014). Natural forms vitamin $E$ : Metabolism, antioxidant, anti-inflammatory activities and their role in disease prevention and therapy. Free Radic. Biol. Med. 72: 76-90.

40) Flora, S. J. (2009). Structural Chemical and biological aspects of antioxidants for strategies against metal and metalloid exposure. Oxid. Med. Cell Longev. 2(4): 191-206.

41) Elaroussi, M. A., Fattah, M. A., Meky, N. H., Ezzat, I. E. and Wakwak, M. M. (2007). Effects of vitamin E, age and sex on performance of Japanese quil. 1. Haematological indices and liver function. Br. Poult. Sci. 48(6): 669-677.

42) Dissayabutra, T., Tosukhowong, P. and Seksan, P. (2005). The benefits of vitamin $C$ and vitamin $E$ in children with beta-thalassemia with high oxidative stress. J. Med. Assoc Thai. 88 Suppl 4: S317-S321.

43) Cherdyntseva, N., Shishkina, A., Butorin I., Murase, H., Gervas, P. and Kagiya, T. V. (2005). Effect of tocopherol-monoglucoside (TMG), a watersoluble glycosylated derivate of vitamin $\mathrm{E}$, on hematopoietic recovery in irradiated mice. J. Radiat. Res. 46(1): 37-41.

44) Brigelius-Flohe, R. (2009). Vitamin E: the shrew waiting to be tamed. Free Radic. Boil. Med. 46(5): 543-554.

45) Jilani, T. and Iqbal, M. P. (2011). Does vitamin E have a role in treatment and prevention of anemia? Pak. J. Pharm. Sci. 24(2): 237-242.

46) Junge, B., Carrion, Y., Bosco, C., Galleano, M., Puntarulo, S., Tapia, G. and Videla, L. A. (2001). Effects of iron overload and lindane intoxication in relation to oxidative stress, Kupffer cell function, and liver injury in the rat. Toxicol. Appl. Pharmacol. 170(1): 23-28. 
47) Zhao, Y., Li, H., Gao, Z. and Xu, H. (2005). Effects of dietary baicalin supplementation on iron overload-induced mouse liver oxidative injury. Eur. J. Pharmacol. 509(2-3): 195-200.

48) Zhang, Y., Li, H., Zhao, Y. and Gao, Z. (2006). Dietary supplementation of baicalin and quercetin attenuates iron overload induced mouse liver injury. Eur. J. Phamacol. 535(1-3): 263-269.

49) Nahdi, A., Hammani, I., Brasse-Lagnel, C., Pilard, N., Hamdaoui, M. H., Beaumont, C. and El May, M. (2010). Influence of garlic or its main active component diallyl disulfide on iron bioavailability and toxicity. Nutr. Res. 30(2): 85-95.

50) Lekawanvijit, S. and Chattipakorn, N. (2009). Iron overload thalassemic cardiomyopathy: iron status assessment and mechanisms of mechanical and electrical disturbance due to iron toxicity. Can. J. Cardiol. 25(4): 213-218.

51) Andrews, N. C. and Schmidt, P. J. (2007). Iron homeostasis. Annu. Rev. physiol. 69: 69-85.

52) Torti, F. M. and Torti, S. V. (2002). Regulation of ferritin genes and protein. Blood. 99(10): 35053516.

53) Lass, A. and Sohal. R. S. (2000). Effect of coenzyme $\mathrm{Q}(10)$ and alpha tocopherol content of mitochondira on the production of superoxide anion radicals. FASEB. J. 14: 87-94.

54) Agrawal, R., Sharma, P. K. and Rao, G. S. (2001). Release of iron from ferritin by metabolites of benzene and superoxide radical generating agents. Toxicology. 168(3): 223-230.

55) Silva, M., Silva, M. E., de Paula, H., Carneiro, C. M. and Pedrosa, M. L. (2008). Iron overload alters glucose homeostasis, causes liver steatosis, and increases serum triacylglycerols in rats. Nutr. Res. 28(6): 391-398.

56) Asare, G. A., Mossanda, K. S., Kew, M. C., Paterson, A. C., Kahler-Venter, C. P. and Siziba, K. (2006). Hepatocellular carcinoma caused by iron overload: a possible mechanism of direct hepatocarcinogenicity. Toxicology 219(1-3): 41-52.

57) Hashemian, Z., Hashemi, A. and Fateminasab, M. (2012). The benefits of vitamin $\mathrm{E}$ on liver function and the hemopoietic System in thalassemia Patients. Iran J. Ped. Hematol. Oncol. 2(4):153-158.

58) Catalgol, B. and Ozer, N. K. (2012). Protective effects of vitamin $\mathrm{E}$ against hypercholesterolemiainduced age-related diseases. Genes Nutr. 7(1): 9198.

59) Achuba, F. I. (2005). Effect of Vitamins $C$ and $E$ intake on blood lipid concentration, lipid peroxidation, superoxide dismutase and catalase activities in rabbit fed petroleum contaminated diet. Pakistan Journal of nutrition 4(5): 330-335.

60) Schimmel, M. and Bauer, G. (2002). Proapoptotic and redox state-related signaling of reactive oxygen species generated by transformed fibroblasts. Oncogene 21(38): 5886-5896.
61) Jomova, K. and Valko, M. (2011). Advances in metal-induced oxidative stress and human disease. Toxicology 283(2-3): 65-87.

62) Fang, Y. Z., Yang, S. and Wu, G. (2002). Free radicals, antioxidants, and nutrition. Nutrition. 18(10): 872-879.

63) Chakraborty, D. and Bhattacharyya, M. (2001). Antioxidant defense status of red blood cells of patients with beta-thalassemia and Ebeta-thalassemia. Clin. Chim. Acta. 305(1-2): 123-129.

64) Valko, M., Rhodes, C. J., Moncol, J., Izakovic, M. and Mazur, M. (2006). Free radicals, metals and antioxidants in oxidative stress-induced cancer. Chem. Biol. Interact. 160(1): 1- 40.

65) Koyu, A., Ozguner, F., Caliskan, S. and Karaca, H. (2005). Preventive effect of vitamin E on ironinduced oxidative damage in rabbit. Toxicol. Ind. Health. 21(9): 239-242.

66) Chen, X., Touyz, R. M., Park, J. B. and Schiffrin, E. (2001). Antioxidant effect of vitamins $C$ and $E$ are associated with altered activation of vascular NADPH oxidase and superoxide dismutase in strokeprone SHR. Hypertension. 38(3 Pt 2): 606-611.

67) Hou, Y. J., Zhao, Y. Y., Xiong, B., Cui, X. S., Kim, N. H., Xu, Y. X. and Sun, S. C. (2013). Mycotoxincontaining diet causes oxidative stress in the mouse. PLoS One. 8(3): e60374.

68) Naithani, R., Chandra, J., Bhattacharjee, J., Verma, P. and Narayan, S. (2006). Peroxidative stress and antioxidant enzymes in children with betathalassemia major. Pediatr. Blood Cancer 46(7): 780785.

69) Hundekari, I. A., Suryakar, A. N. and Rathi, D. B. (2013). Acute organo-phosphorus pesticide poisoning in North Karnataka, India: oxidative damage, haemoglobin level and total leukocyte. Afr. Health Sci. 13(1): 129-136.

70) Ranjbar, A., Solhi, H., Mashayekhi, F. J., Susanabdi, A., Rezaie, A. and Abdollahi, M. (2005). Oxidative stress in acute human poisoning with organophosphorus insecticides; a case control study. Environ. Toxicol. Pharmacol. 20(1): 88-91.

71) Casaril, M., Stanzial, A. M., Tognella, P., Pantalena, M., Capra F, Colombari $R$ and Corrocher R. (2000). Role of iron load on fibrogenesis in chronic hepatitis C. Hepatogastroenterology. 47(31): 220-225.

72) Kolios, G., Valatas, V. and Kouroumalis, E. (2006). Role of Kupffer cells in the pathogenesis of liver disease. World. J. Gastroenterol. 12(46): 74137420.

73) Arezzini, B., Lunghi, B., Lungarella, G. and Gradi, C. (2003). Iron overload enhances the development of experimental liver cirrhosis in mice. Int. J. Biochem. Cell. Biol. 35(4): 486-495. 